Check for updates

Cite this: RSC Adv., 2021, 11, 3399

Received 12th October 2020

Accepted 14th December 2020

DOI: $10.1039 / \mathrm{d} 0 \mathrm{ra0} 8672 \mathrm{j}$

rsc.li/rsc-advances

\section{Thriving artificial underwater drag-reduction materials inspired from aquatic animals: progresses and challenges}

\author{
Guizhong Tian, Dongliang Fan, Xiaoming Feng and Honggen Zhou (DD *
}

In the past decades, drag-reduction surfaces have attracted more and more attention due to their potentiality and wide applications in various fields such as traffic, energy transportation, agriculture, textile industry, and military. However, there are still some drag-reduction materials that need to be deeply explored. Fortunately, natural creatures always have the best properties after long-term evolution; aquatic organisms have diversified surface microstructures and drag-reducing materials, which provide design templates for the development of thriving artificial underwater drag-reduction materials. Aquatic animals are tamed by the current while fighting against the water, and thus have excellent drag reduction that is unparalleled in water. Inspired by biological principles, using aquatic animals as a bionic object to develop and reduce frictional resistance in fluids has attracted more attention in the past few years. More and more aquatic animals bring new inspiration for drag-reduction surfaces and a tremendous amount of research effort has been put into the study of surface dragreduction, with an aim to seek the surface structure with the best drag-reduction effect and explore the drag-reduction mechanism. This present paper reviews the research on drag-reduction surfaces inspired by aquatic animals, including sharks, dolphins, and other aquatic animals. Aquatic animals as bionic objects are described in detail, with a discussion on the drag-reduction mechanism and drag-reduction effect to understand the development of underwater drag-reduction fully. In bionic manufacturing, the effective combination of various preparation methods is summarized. Moreover, bionic surfaces are briefly explained in terms of traffic, energy sources, sports, and agriculture. In the end, both existing problems in bionic research and future research prospects are proposed. This paper may provide a better and more comprehensive understanding of the current research status of aquatic animalsinspired drag reduction.

\section{Introduction}

With the aggravation of the global energy crisis, the research on underwater drag reduction and performance improvement technology is becoming increasingly urgent. Oceans are home to countless aquatic organisms, which are considered as the origin of life. Furthermore, after 3.7 billion years of natural selection and evolution of aquatic species, creatures living underwater are characterized by efficient energy use and lowdrag activities. Therefore, they have the best answers when we seek to improve or optimize underwater drag-reduction materials. Fortunately, the application of bionics allows us to imitate biological materials in order to manufacture and process dragreducing materials. Aquatic organisms survive in water as they have super adaptability to resist the impact and friction of water currents. In the long biological evolution, sophisticated body

College of Mechanical Engineering, Jiangsu Provincial Key Laboratory of Advanced Manufacturing for Marine Mechanical Equipment, Jiangsu University of Science and Technology, Zhenjiang, P. R. China.E-mail: justtgz@163.com structure and a characteristic way of motion have been developed to combat the complex environment. There are many types of aquatic organisms, including aquatic bacteria, aquatic fungi, aquatic plants, and aquatic animals. Among them, aquatic animals represented by vertebrates have taken the lead in this long-term evolution. For example, fish are the oldest vertebrates that inhabit almost all marine environments on Earth from freshwater lakes and rivers to saltwater seas and oceans. They have a wide variety of complex species (more than 36000 living fish species in the world) and their powerful ability to adapt to the environment is widely known.

As we know, the living environment of most aquatic animals is liquid water medium for physiological activities. The interface between the water environment and the body surface is crucial for both the physiology and hydrodynamic functions of aquatic animals in water. Oxygen, ions, and carbon dioxide exchange occurs around the surface of the aquatic animals' body tissues to maintain the basic physiological activities. ${ }^{1}$ For the hydrodynamic functions of aquatic animals, surface texture and body structure have potential mechanisms for drag 
reduction. ${ }^{\mathbf{1 , 2}}$ Aquatic animals need to consume the most energy to overcome surface resistance during underwater navigation and transportation, and have the function of drag reduction in long evolution and natural selection owing to the physical challenge of motion in water.

The countermeasures are rough as follows: (1) friction drag and viscous drag caused by direct contact of the skin surface with fluids. Different kinds of aquatic animals have different special responses to overcome these obstacles, such as shark's scales and dolphin's flexible compliant skin. ${ }^{3}$ Furthermore, mucus covering the surface texture of aquatic animals gives a slippery feeling, which has always been considered as an indispensable factor in reducing the frictional drag and viscous drag of aquatic animals. Tian et al. utilized the DSD/SST method to simulate and propose that mucus also has the effect of enhancing thrust and reducing noise. ${ }^{4}$ The combination of scales and mucus on aquatic animals' skin protects them from being scratched by sharp objects underwater and swims and wears in the water for a long time, resists parasites, and plays a clean and antifouling effect. (2) The pressure drag exists between the head and the tail. In general, the streamline of the aquatic animals' body greatly reduces the fluid pressure drag due to the weakening of the negative vortex effect or avoiding the formation of negative vortices. Research has indicated that shark scales and flexible skin seem to be able to reduce pressure drag through passive flow control mechanisms. The skin and streamlined body control the vortex to increase the fluid velocity in the boundary layer, thereby reducing separation and thus reducing pressure drag. ${ }^{3,5}$ Aquatic animals not only passively reduce drag in the streamline and interface performance but also have an amazing cruise strategy during active swimming.

With the expansion of marine operations and exploration, the technical improvement of underwater drag reduction is necessary. Aquatic animals have been used as a source of inspiration for many vehicles, such as ships, aviation, submarines, and underwater operating equipment. ${ }^{6}$ The main reasons are their adaptability to environmental changes underwater and the ability to self-heal/correct the system. ${ }^{7}$ Cruising aquatic animals can utilize the surrounding environment to save energy. Also, the energy consumption of fish organisms in water is different from common sense. ${ }^{8}$ During the active motion of aquatic animals, they accelerate intermittently, then constantly maintain the cruise speed, and alternately climb and sink forward.9,10 Moreover, biological aquatic animals are "stingy" with their energy while adapting to underwater activities and their cruising strategy is to spend the least energy to reach the maximum distance of sailing from an evolutionary perspective. Compared to large-sized sharks and dolphins, small-sized aquatic animals seem to have insufficient energy to resist turbulent water currents and are eliminated by the environment. On the contrary, Fletcher et al. explained why some smaller fish (flatfish) could maintain stable motion in turbulent river water. Small-sized fish utilize a non-smooth riverbed base with a low-velocity thin layer cruising strategy, which is close to the river bottom, to reduce their energy consumption. ${ }^{11}$ In the experiment, aquatic animals utilized less oxygen in highturbulence compared with low-turbulence flow at medium and high swimming speeds, i.e., energy consumption is weakened. ${ }^{8}$ Aquatic animals not only utilize the turbulent flow state but also borrow the disturbance generated by other large moving objects, which changes their body motion by staying behind the hull and 'synchronize' with the current vortex, thereby reducing energy consumption. This behavior is called Karman gaiting: ,12,13 $^{\mathbf{1}}$ Passive drag reduction and adaptive active movement provide survival advantages for aquatic animals, also bringing new ideas and inspiration to different fields of research.

In recent years, with the discovery of other biomorphic phenomena at different scales, the traditional view of drag reduction on smooth surfaces has been questioned, while the bionic surface of drag reduction inspired by aquatic animals has been extensively studied and experimented. Surface drag reduction emphasizes the actual value in applications, including traffic, energy transportation, agriculture, textile industry, military, sport, and industry. So far, there have been many research results of bionic drag reduction from aquatic animals as bionic prototypes, which can be roughly divided into three categories: sharks, dolphins, and other aquatic animals.

Until now, some review articles on bio-inspired drag-reducing surface have been published. ${ }^{14-17}$ However, the discussions focusing on the development of the aquatic animals-inspired dragreduction surfaces are relatively rare. In this review, drag-reduction biological prototypes represented by sharks and dolphins, the latest signs of progress related to biology, drag-reduction surfaces, drag-reduction mechanisms, manufacturing, and applications are summarized. In this review, we aim to discuss the recent developments in the thriving artificial drag-reduction materials inspired by aquatic animals. This review is mainly composed of five sections. Section 2 presents the drag-reduction surfaces inspired by aquatic animals in detail and summarized their drag-reduction mechanism. In the following Section 3, some typical bionic dragreduction surface processing and manufacturing methods are described. Then, in Section 4, the applications of bionic dragreduction surfaces in practical engineering are briefly described. In the final Section 5, the problems and development direction of future research on bionic drag-reduction surfaces are proposed and addressed. This review may provide a better comprehension of the current research status of aquatic animals-inspired drag reduction surfaces.

\section{Biological prototypes}

In the process of fighting against the natural environment, organisms have evolved to break the constraints of their structure and biological prototypes with drag-reducing surfaces have emerged. In recent years, researchers have selected suitable biological prototypes, as shown in Table 1, to screen various surfaces with drag-reduction properties. In this chapter, sharks, dolphins, and other aquatic animals are used as bionic prototypes to review their drag-reduction surfaces and drag-reduction mechanisms.

\subsection{Shark-inspired drag reduction}

Sharks have attracted the most attention as deep-sea predators because strong bursts and long cruises that they have evolved 

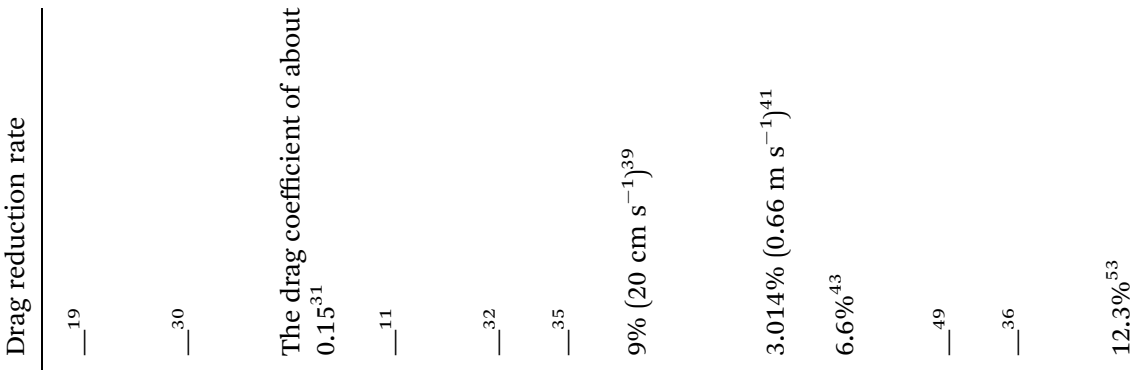

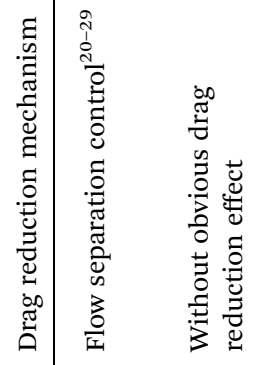
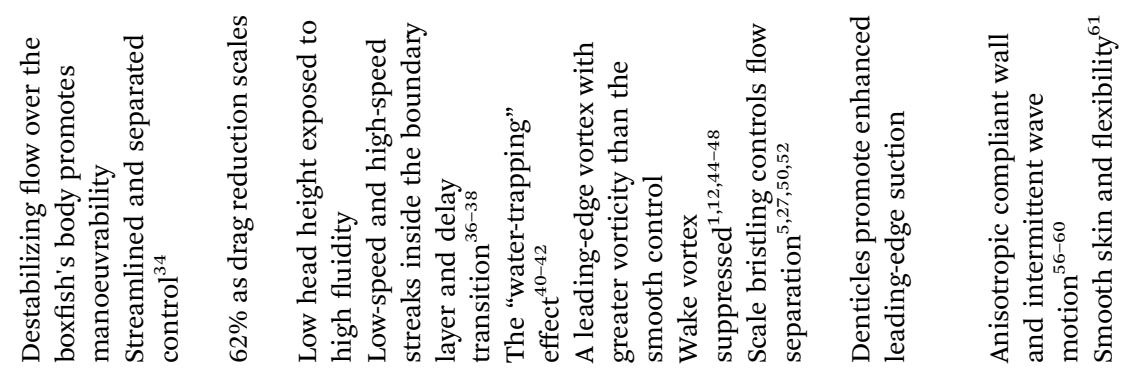

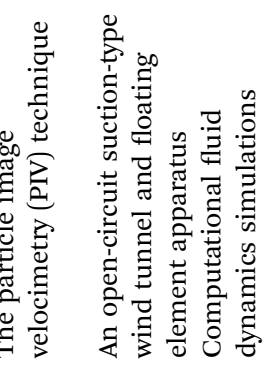
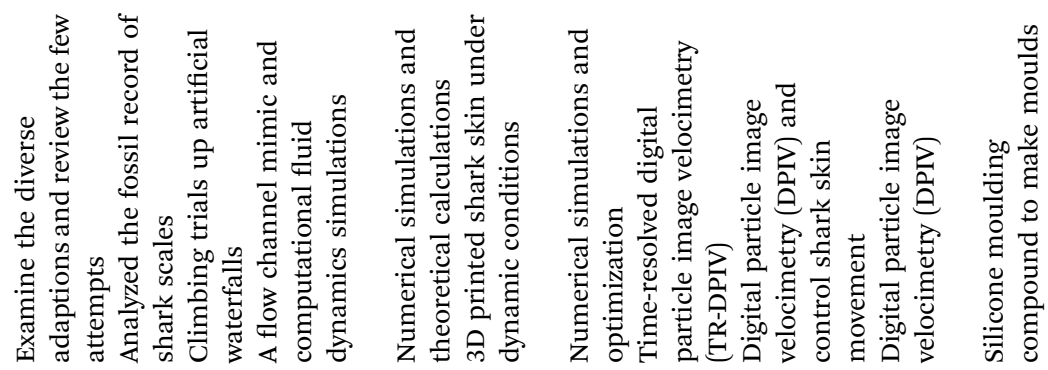

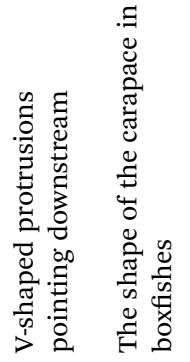<smiles>CC1[C@@H](C)[C@@H](C)C[C@H](C)[C@@H]1C</smiles>

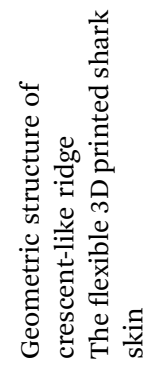

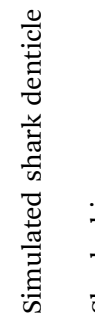

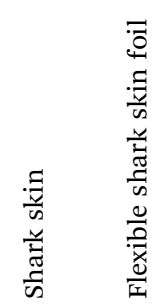

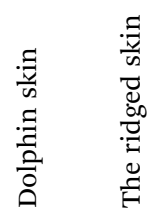
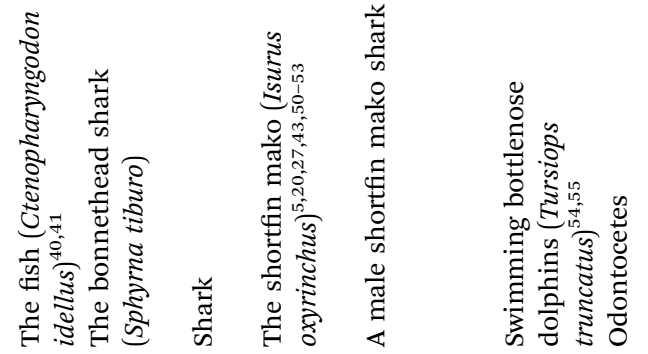


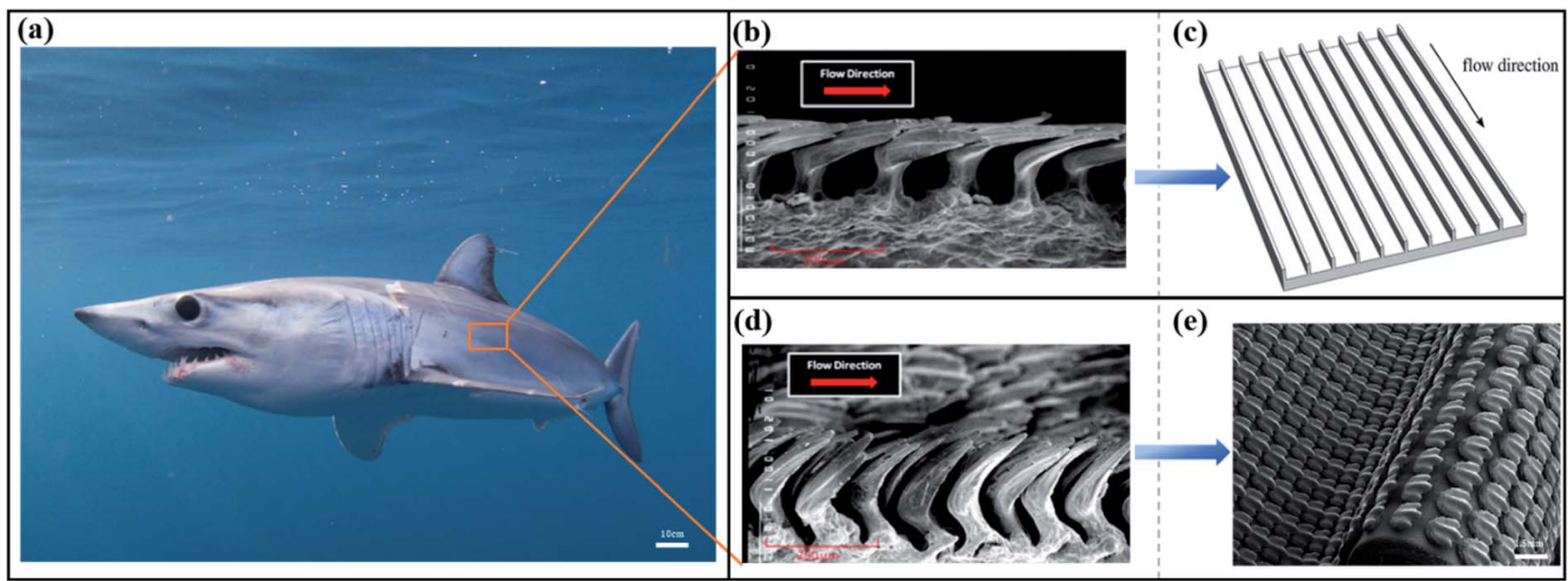

Fig. 1 (a) The Mako shark. (b) SEM of the non-bristled scales is replicated into riblets. Reproduced from ref. 5 with permission from IOP Publishing, copyright 2017. (c) Schematic picture of the riblets. Reproduced from ref. 50 with permission from the Royal Society, copyright 2018. (d) SEM of the bristled scales is replicated into denticles and attached to the bionic surface of the flexible substrate. Reproduced from ref. 5 with permission from IOP Publishing, copyright 2017. (e) Schematic picture of the denticles with 3D printing. Reproduced from ref. 43 with permission from the Company of Biologists Ltd, copyright 2014.

after a long period of natural selection. A suite of adaptations has been honed and extensively explored to reduce energy expenditure. Consequently, to reduce pressure drag due to a difference in the pressure around the body, sharks, like most fish, have evolved streamlined structures. The gills of sharks are believed to be the main respiratory organ, which is found to reduce the drag due to lift. Also, skin drag due to boundary layer formation can be reduced by shark skin. Among the many ways of reducing drag, the excellent drag reduction ability of shark skin has been extensively studied. Many thousands of minute tooth-like scales (as shown in Fig. 1), called dermal denticles or placoid scales, are covered over the shark's surface, which has been assumed to play an important role in locomotion. Nearly 450 species of shark and different parts of shark individuals have covered other characteristics of the denticles to adapt to the changing flow field in the ocean. ${ }^{63}$ Therefore, there is a challenge in accurate skin surface imaging as it is difficult to find a uniform way to characterize the shark's skin surface. ${ }^{2}$

However, regular grooves with triangular or rectangular cross-sections, called riblets, are utilized to simplify the dermal denticles of shark's surface (Fig. 1(b)) and these replicate riblets are also subjected to extensive hydrodynamic and aerodynamic tests to explain the drag-reduction mechanisms. Initially, the surface imaging technology of fish skin has been constantly developed, such as scanning electron microscopy (SEM), ${ }^{64}$ microcomputed tomography $(\mu \mathrm{CT})$, gel-based surface profilometry, and histology ${ }^{65}$ based on which models of real shark denticles have been recreated and tested. Besides, numerical simulation has also been widely used to better understand the flow field around shark skin. The two forms of replicating the shark's skin surface are described in the following paragraphs, namely, riblets and denticles.

\subsubsection{Riblets}

2.1.1.1. Morphology. Most of the inspiration of non-smooth surfaces comes from shark skin, which possesses special drag- reducing properties in water. Sharkskin has been simplified into a ' $2 \mathrm{D}$ ' riblets shape to analyze its drag reduction, which breaks through the tradition of smoother drag. An extensive review of the rough-wall turbulent boundary layer is discussed. ${ }^{17,66,67}$ Riblets are textures applied to the surface and are usually grooves aligned with the flow direction. A riblet is believed to have flow guidance and promotes anisotropic flow and effectively controls the naturally-occurring turbulent vortex, thereby reducing the capacity exchange and shear stress to the boundary layer, locking vortices generated in the viscous sublayer inside the groove to achieve drag reduction. ${ }^{68} \mathrm{~A}$ recent review of riblets was provided by Martin and Bhushan to optimize the riblet dimensions in many ways to get the best riblet parameters for drag, ${ }^{69}$ as shown in Fig. 2.

Considering different riblet geometry and flow channel conditions, a better experimental comparison can be made and the dimensionless parameters (denoted by the + symbol) in terms of wall surface are summarized. The riblet spacing $(s)$, height $(h)$, thickness $(t)$, and boundary layer thickness $(\delta)$ are defined as:

$$
\begin{gathered}
s^{+}=s / l_{v} \\
h^{+}=h / l_{v} \\
t^{+}=t / l_{v} \\
\delta^{+}=\delta / l_{v}
\end{gathered}
$$

The wall unit $l_{v}$ is defined as $l_{v}=v / u_{\tau}$, with $u_{\tau}$, the friction velocity, $u_{\tau}=\sqrt{\tau_{\mathrm{w}} / \rho}, \tau_{\mathrm{w}}$ being the total stress at the rough wall, $\rho$ as the density of the fluid, and $v$ is the kinematic viscosity of the liquid.

Velocity distribution in the logarithmic layer in the boundary layer is found to follow the law of logarithmic arrangement; thus, it is utilized to characterize the friction velocity of the boundary layer. In smooth pipes, the universal logarithmic law is written as 

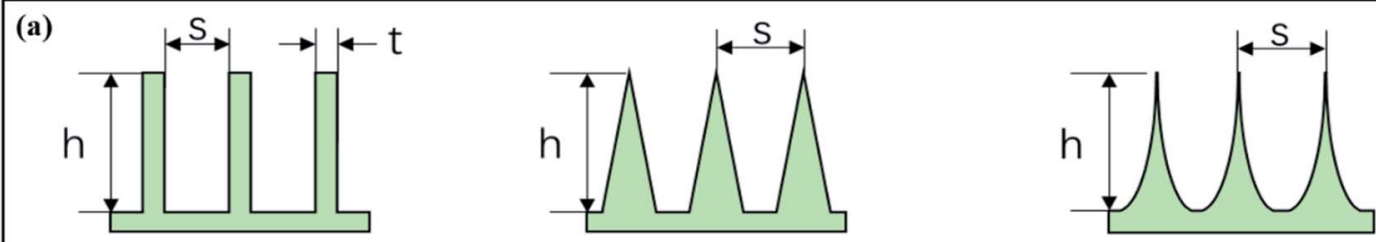

\section{Blade}

Sawtooth

Scalloped
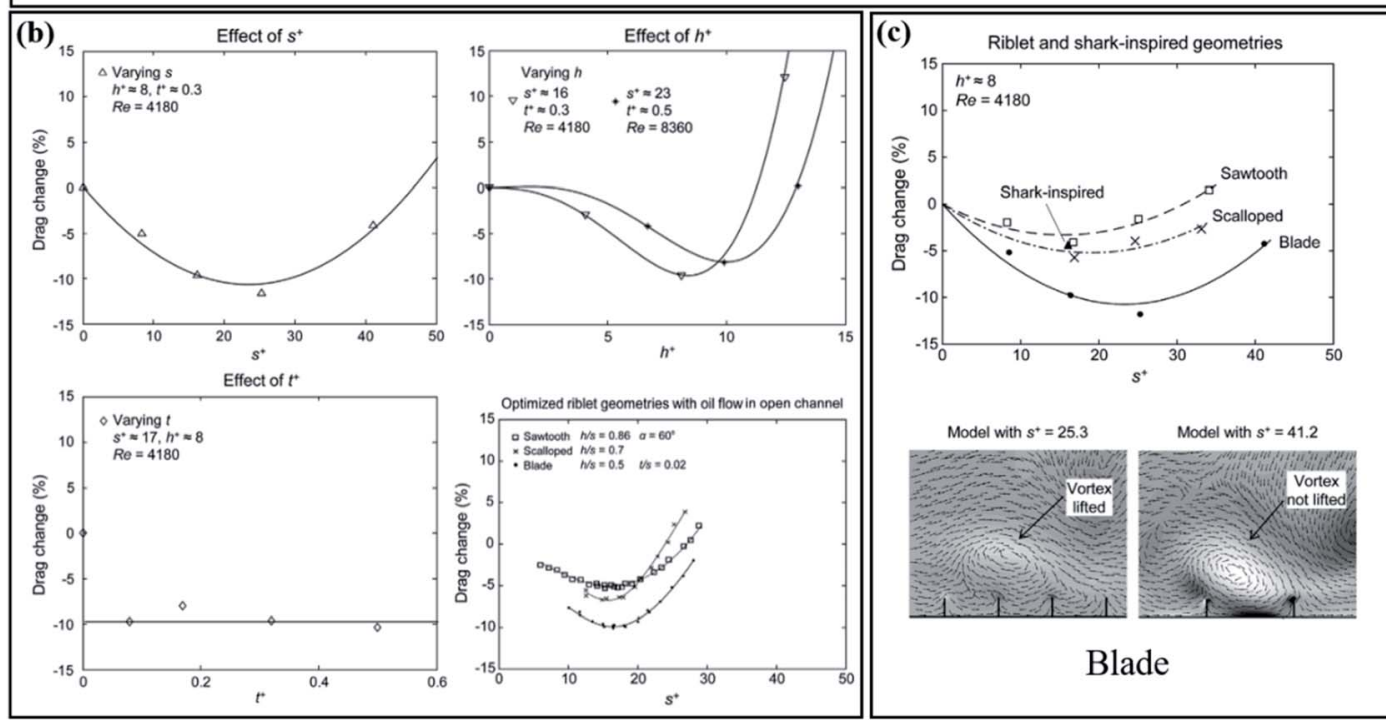

Fig. 2 (a) The typical cross-sectional geometries of riblets with' blade', 'sawtooth', and 'scalloped' geometries. (b) Drag change rate as a function of the dimensionless parameters of riblets spacing $(s)$, height $(h)$, and thickness $(t)$. The spacing and height of the riblets are the keys to the drag reduction rate, while the thickness does not seem to have any effect. The optimal spacing $s^{+}=15-20$, height $h^{+} \approx 8-10$, and thickness $t^{+}=$ small. Compared with the oil fluid medium, the height and spacing have a strong correlation with the drag reduction rate. The optimal ratio of spacing and height gives a ratio of $h / s \approx 0.5$. Reproduced from ref. 74 with permission from Cambridge University Press, copyright 2014. (c) Blade geometry shows the greatest drag reduction (11.6\%). The optimization of riblet spacing $\left(s^{+}=25.3\right)$ makes the large-scale vortex lift from the wall and the small-scale vortex acts on the groove to reduce the resistance. The increase in the spacing $\left(s^{+}=41.2\right)$ makes the large vortex 'slide down' into the inner wall. Reproduced from ref. 69 with permission from Academic Press Inc., copyright 2016.

$$
U^{+}=\frac{1}{\kappa} \ln y^{+}+A
$$

with the von Kármán constant $\kappa=0.392$ and $A=4.48 .^{70}$ The dimensionless wall-normal distance $y^{+}$and the mean velocity $U^{+}$is defined as $y^{+}=y / l_{v}, U^{+}=U / u_{\tau}$. Also, there is a general difference between the experiment and numerical simulation, and this difference can be corrected. ${ }^{70}$ When the surface is a non-smooth surface, a downward shift in the inner-normalized mean velocity is often experienced, ${ }^{71,72}$ which is called the roughness function $\Delta U^{+}$; the log law of flow on a rough surface is written as

$$
U^{+}=\frac{1}{\kappa} \ln y^{+}+A-\Delta U^{+}
$$

Using the rough function $\Delta U^{+}$can intuitively express the drag reduction effect of the groove surface relative to the smooth surface. ${ }^{73}$ When $\Delta U^{+}$is positive, the velocity distribution shifts upwards and the drag is less than the corresponding smooth wall. Conversely, when $\Delta U^{+}$is negative, the riblet is considered to have a drag reduction function and its size is utilized to evaluate the drag reduction effect. ${ }^{71}$ The bionic riblets are optimized and described because of their implied excellent drag reduction performance. In this process, why a non-smooth surface, such as a groove, reduces drag compared to the smooth surface is explored and discussed by researchers. A more profound understanding of the role and function of these surfaces as well as various drag-reduction mechanisms are discussed in the next section.

2.1.1.2. Mechanisms. The role of riblets in drag reduction is of great concern and numerous achievements have been obtained. ${ }^{2,75}$ Initially, the riblets of shark skin increase the area of the wetted surface. This structure has been supposed to be not conducive for drag reduction. Smooth surfaces are the most used as comparisons of non-smooth surfaces. Also, the foundation of riblet research is to establish and evolve from smooth surfaces. Therefore, understanding the boundary layer of smooth surfaces is beneficial for the further exploration of nonsmooth surfaces. As shown as Fig. 3(a), the boundary layer develops from $x=0$ and its average velocity is less than the flow velocity $U_{\mathrm{e}}$; the viscous sublayer, buffer layer, logarithmic layer, and wake layer make up the structure of the boundary layer.

The logarithmic layers of smooth surfaces and riblets are defined by eqn (5) and (6). On a practical level, the cross-stream translation of the streamwise vortices is impeded by the small 


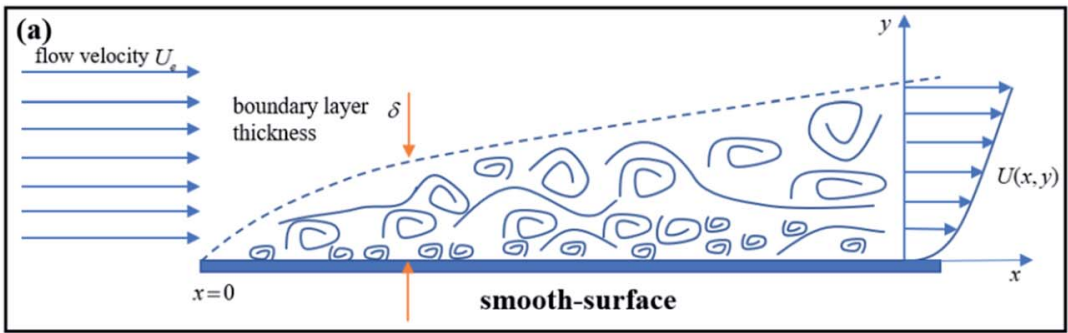

(c)
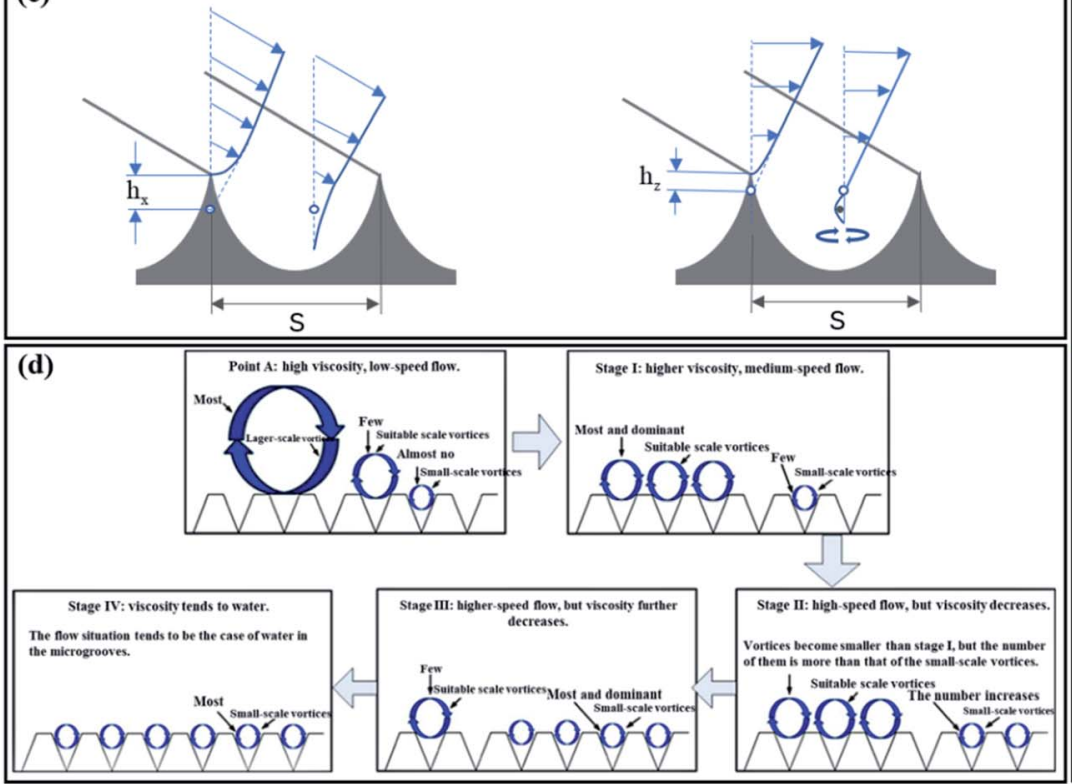

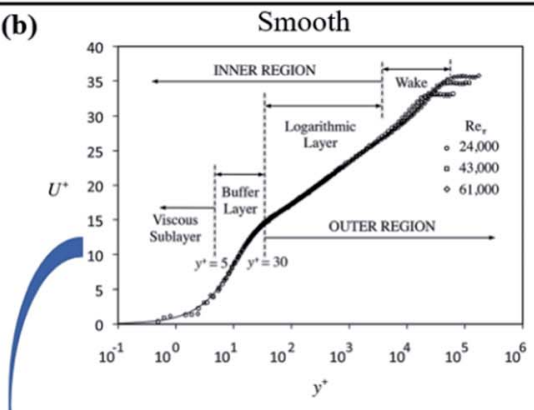

Viscous sublayer: $0<y^{+}<5 \quad U^{+}=y^{+}$

Buffer layer: $\quad 5<y^{+}<30 \quad U^{+}=f_{1}\left(y^{+}\right)$

Logarithmic layer: $30<y^{+}<0.2 \delta^{+} U^{+}=\ln \left(y^{+}\right) / k+B$

Wake layer: $\quad 0.2 \delta^{+}<y^{+} \quad U_{e}-U=f_{2}(y / \delta)$

(e)

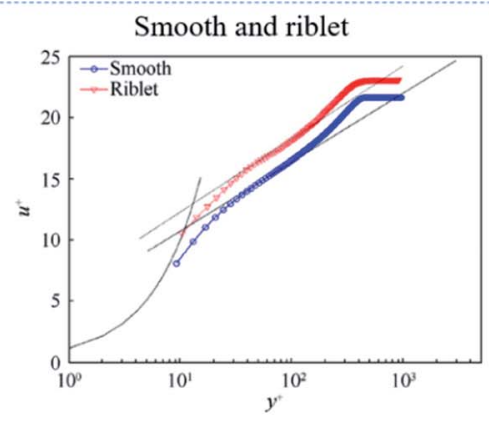

Fig. 3 (a) Flat boundary layer structure. $x=0$ as the starting point for the development of the boundary layer. Reproduced from ref. 17 with permission from American Society of Mechanical Engineers (ASME), copyright 2016. (b) Regional division and functional representation of the mean velocity profile of a smooth-flat-plate boundary layer. Reproduced from ref. 17 with permission from American Society of Mechanical Engineers (ASME), copyright 2016. (c) The schematic diagram of the velocity profile along the flow direction (left) and the span direction (right) at the crest and trough of the riblet wall. A hollow circle represents the virtual origin. Reproduced from ref. 78 with permission from Cambridge University Press, copyright 2019. (d) The evolution from large-scale vortex to a small-scale vortex. Reproduced from ref. 79 with permission from Elsevier BV, copyright 2016. (e) Comparison of average velocity profile in the boundary layer between the smooth plate and riblet. Reproduced from ref. 73 with permission from Chinese Journal of Aeronautics, copyright 2019.

riblets that cover the surface of the sharks. This directly affects the thickening of the boundary layer and the Reynolds shear stress decreased; thus, drag reduction reduced. However, the mechanism of riblet surface drag-reduction in the turbulent boundary layer is complex and the entirety of the phenomena is not yet fully explained. In theoretical research, an explanation of the drag-reduction mechanism of riblets in laminar flow conditions goes by the name of 'the protruding height theory' in the fluid mechanics' community, which has been known for a long time. ${ }^{15,76}$ As the riblets protrude into the flow field, for protrusions smaller than $y^{+}=5$ (Fig. 3(b)), viscous flow theory can be used to calculate the flow around very small riblets. ${ }^{77}$

The drag increases instead of decreasing due to the interaction between riblets and vortexes. The reason for drag reduction is that the presence of vortexes increases the thickness of the viscosity underlying layer, so the flow tends to more stable and momentum exchange is suppressed. As opposed to boundary-layer growth, drag reduction was evaluated more quantitatively by the protruding height theory. The velocity gradient has been used to calculate the average velocity at different heights of the laminar flow area on the surface of the riblet and they are compared with that of the plate flow. For laminar flow, the effective streamwise protrusion height $h$ has been defined as the distance between the apparent origin and the riblet tip (Fig. 3(c)) to determine how far the riblet tips protrude into the boundary layer. In cross-stream, the virtual origin is closer to the riblet tips than for longitudinal flow. The effective cross-stream protrusion height, $h_{z}$, can also be defined in the same way. For any riblet geometry, $h_{x}-h_{z}$ has been proposed to express the measure that the riblet geometry reduces the transverse vortex under low Reynolds number flows. ${ }^{22}$ It is more difficult to laterally move than move longitudinally in the riblet when differences between cross-stream protrusion height and streamwise protrusion height $\left(h_{x}-h_{z}\right)$ exist. In this way, momentum transfer and shear stress are reduced by hampering the crossflow.

The occurrence of vortex ejection into the outer boundary layers can be reduced by impeding the cross-stream translation of the 
streamwise vortices, and thereby, momentum exchange is suppressed. High-speed flow lumps approaching the surface into the low-speed regions, called sweeps, and low-speed flow moving away from the surface into the high-speed regions of the flow, called ejections, constitute the strong momentum exchange in the turbulent boundary layer. ${ }^{80}$ Yang et al. supposed that the riblets directly affect the ejection (Q2) and sweep (Q4) events near the wall, especially the Q4 events, which reduced the amplitude of the spanwise vorticity, ${ }^{\mathbf{1}}$ thereby dampening the momentum exchange between the proximal and outer regions of the turbulent boundary layer to reduce the drag (Fig. 3(d)).

Vortices that are formed on the ridge surface play a crucial role. Higher velocity vortices are distributed at the top of the riblets and this localized area experiences high-shear stresses. Low-shear stresses are produced in the valleys of the riblets. However, the vortices split into some secondary vortex that entered the riblet valleys transiently. This secondary vortex, such as 'bearing', effectively weakens the ability of momentum exchange and the event of ejecting low-speed flow is diminished. Also, the 'bearing' has been interpreted to change sliding friction into rolling friction with less drag. The vortex is embedded so that the fluid passes through the surface and produces partial slippage, thereby leading to higher momentum adjacent to the surface. ${ }^{82,83}$ It also plays a role in the stable boundary layer and reduces drag between the fluid and the wall. This explanation has been later corroborated. ${ }^{84}$ The suppression of lateral fluctuations and the capture of small-scale vortices are used to explain the drag reduction of the riblets; ${ }^{85}$ also, the complexity of the shark scales is considered to be due to the simplified riblet only, which implied drag reduction mechanism; thus, real and highly similar shark skins, whose surface is covered by denticles, have also been hydrodynamically tested to explain the reason for shark skin drag reduction.

\subsubsection{Denticles}

2.1.2.1. Morphology. As opposed to prototypical riblets, the elastic skin of a shark can also bend and fold while swimming, and shark denticles that stick out of the skin are tooth-like (Fig. 4(a)) with enamelled and dentine outer layers, inner pulp cavity with nerves and blood vessels, and a special structure is composed of the outer crown, neck, and the expanded base imbedded into the dermis. ${ }^{65,86,87}$ The denticles that cover the skin of sharks have unique functional morphotypes, such as drag reduction (Fig. 4(b)), abrasion strength, defence, generalized functions, and ridged abrasion strength. ${ }^{33}$

Large diversities exist in the density of denticles, morphology, size, shape, and direction along the body of the shark (Fig. 4(c)). The different topography on the shark's skin seems to imply the shark's superior hydrodynamic function.
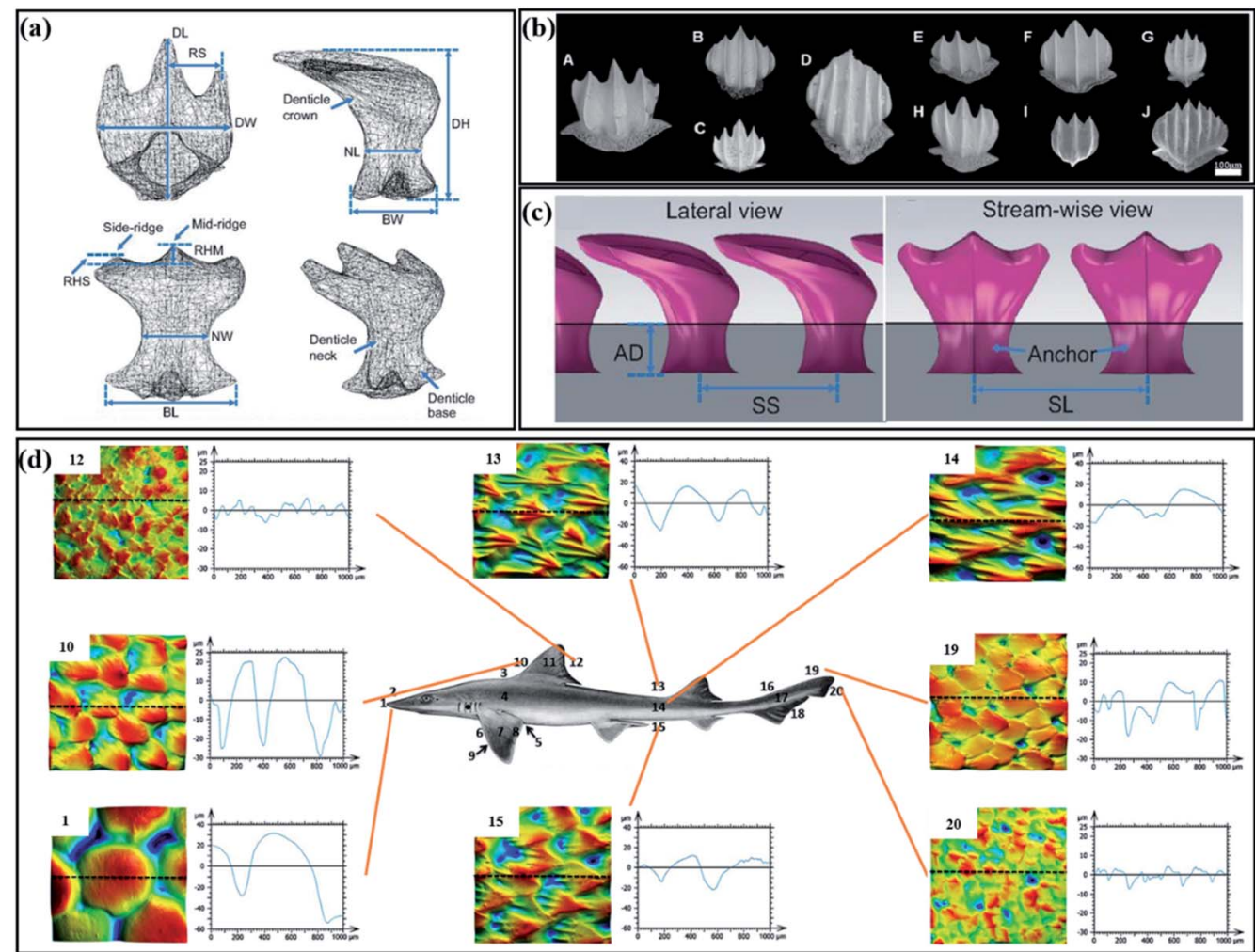

Fig. 4 (a) Three-dimensional reconstructed micro-CT denticle model of a mako shark (Isurus oxyrinchus). Reproduced from ref. 43 with permission from Company of Biologists Ltd, copyright 2014. (b) Scanning electron microscopy (SEM) image of dermal dentin with drag reduction. Reproduced from ref. 33 with permission from Inter-Research, copyright 2017. (c) Parameters of denticles arranged linearly on the membrane substrate. The depth of the anchor (AD), the spacing between two adjacent denticles along the stream-wise direction (SS), and the spacing between two adjacent denticles along the lateral direction (SL). Reproduced from ref. 43 with permission from Inter-Research, copyright 2014. (d) Surface profilometry images from shark skin in different parts. Reproduced from ref. 65 with permission from John Wiley and Sons Inc., copyright 2018. 
The distribution of shark denticles has been studied to show a higher density on the dorsal and ventral sides of the body and the highest angular deviations were discovered by Díez, Soto, and Blanco. ${ }^{51}$ The details concerning the effect of denticle density on the skin stiffness and toughness can be found in the paper by Creager and Porter. ${ }^{88}$ By measuring the mechanical properties of young sharks' skin in different areas, the paper proposed that the denticle density increases the stiffness while decreasing the toughness; however, the ultimate tensile strength is not affected. Another quantified shark skin has been discussed by Ankhelyi et al., ${ }^{65}$ who quantified the surface roughness of the shark surface and reconstructed the threedimensional (3D) denticle structure by micro-CT scanning, gel-based surface profilometry, and histology (Fig. 4(d)). The sharkskin of different parts has different functions. Also, the reason for the unevenness of shark skin is believed to be to resist water erosion and increase the swimming speed. Therefore, the potential hydrodynamic drag-reduction mechanism of shark teeth is introduced in the next chapter.

2.1.2.2. Mechanisms. Non-bristled shake denticles have been modelled as riblets. The hydrodynamic effects of riblets at various scales were studied intensively at an early stage. However, man-made riblets are far less capable of reducing drag than real shark skins. This is because the shark is covered with flexible denticles that can passively adapt to the current (Fig. 5(b)). Also, flexible shark denticles have also been known to bristle up to $50^{\circ}$ in the flank regions. ${ }^{27}$ With this, the real shark skin is placed in a simulated environment for further experiments to explore the drag-reduction mechanism.

Previous research has shown that the increase in the thrust force appears to be related to the existence of a strong leadingedge vortex attached closely to the foil surface. ${ }^{53}$ By comparing the rigid plate foil and the flexible foil with the denticles sanded off the dermis by a mechanical slapping device that mimics the movement of a real shark, Oeffner and Lauder explain that the leading of the vortex is closer to the real shark skin. Thus, skin denticles play a role in promoting enhanced suction of the leading of vortexes to increase the swimming speed. ${ }^{53}$ The leading of vortex is of benefit to shark swimming, which had been confirmed later. ${ }^{43}$ Compared with the smooth control model, the 3D-printed shark skin has better vorticity at the leading of the vortex; thus, the swimming speed is increased and the energy consumption is reduced. Also, when the denticles' periodicity exceeds 13 (expressed in-wall units), this behavior becomes consistent with the riblets, resulting in an increase in the drag. On this basis, the effect of denticle spacing
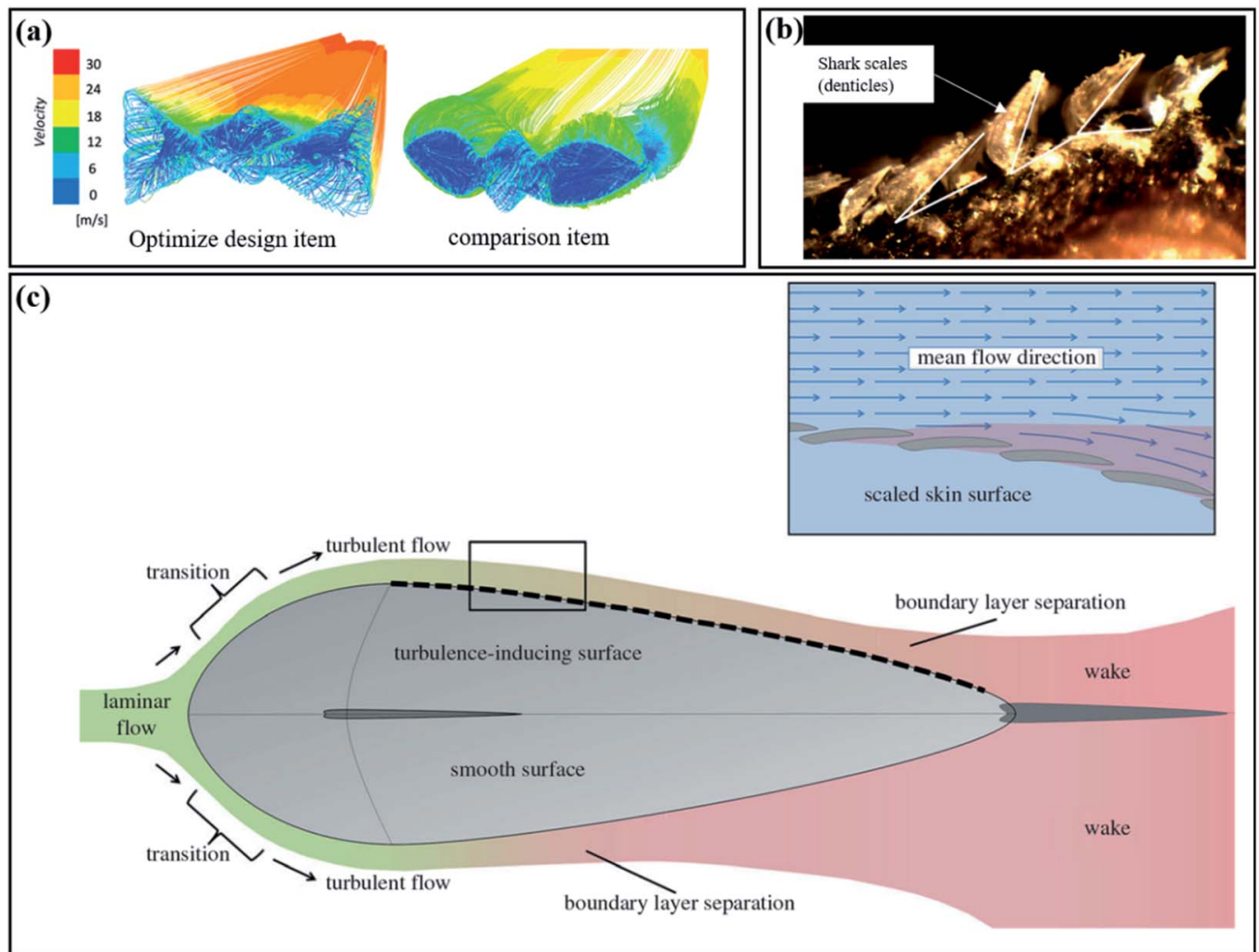

Fig. 5 (a) Optimization of shark single denticle. The flow velocity of the optimized denticle in the wake area is more uniform. Also, the denticles act as vortex generators to energize the boundary layer to reduce the drag. Reproduced from ref. 89 with permission from IOP Publishing Ltd, copyright 2019. (b) Flexible shark skin and bristled shark denticles (magnified 40 times). Reproduced from ref. 36 with permission from Marine Technology Society Inc., copyright 2008. (c) The evolution of fluid in the fish-spindle form. Laminar flow to turbulent flow and then to boundary layer separation to form a wake area. Comparing the non-smooth surface on the axis with the smooth surface under the axis, it has been found that the denticles control the separation of the boundary layer to decrease the wake area, thereby reducing the pressure drag. Reproduced from ref. 11 with permission from the Royal Society, copyright 2014. 
and adjacent denticle arrangement has been studied. ${ }^{90}$ The staggered-overlapped pattern of shark denticles is considered to be more conducive for improve the swimming performance. Later, the denticle size and swimming speed dramatically reflect onto the hydrodynamic effect of the shark denticle, as shown experimentally, which concluded that smaller denticles are beneficial for drag reduction at a higher speed. ${ }^{63}$ Consequently, the vortex generated by shark denticles may play an important role in the drag reduction of active shark navigation. ${ }^{91}$

Bristled shark denticles can be used as vortex generators to control flow separation. The mechanism of flow separation controlled by a vortex generator is different from that controlled by a traditional vortex generator. The design of shark denticlebioinspired structures for aerodynamics are discussed..$^{46,49}$ Shark denticles are regarded as vortex generators to control flow separation in energizing the boundary layer by numerical simulation. The size, shape, and orientation of the denticles are all factors that affect the boundary layer to reduce the drag. The passive mechanism for controlling flow separation has been provided by vortex generators. ${ }^{52}$ Denticles are supposed to bristle passively in the backflow region near or downstream of the separation point. Research demonstrates that denticles play a similar role as special vortex generators in energizing the boundary layer to control flow separation. When the flow meets vortex generators, streamwise vortexes are generated in the downstream and high momentum turbulent energy is mixed in the boundary layer. A large number of vortexes act on the denticle structure to reduce drag.

Research has shown that these particular features of shark skin can reduce drag through passive flow control mechanisms. ${ }^{36}$ Passive flow separates are utilized to explain the drag reduction mechanism of shark denticles and the drag is reduced by delaying the transition from laminar to turbulent flow. Passive flow separation driven by the bristling of this flexibility of individual denticles has been studied. Pressure drag occurs when the flow separates. Also, flow separation can be controlled by the bristling capability of flexible denticles, thereby reducing the pressure drag in the opposite direction of the body movement. ${ }^{20}$ Also, the most elastic denticles are located on the side of the shark body and downstream of the gills, which have flow separation control. The principle of separation control of shark denticles under various strengths of adverse pressure gradient is explained by Afroz et al. The passive bristling of shark denticles is beneficial for drag reduction. ${ }^{5}$

The reason for the passive bristling of shark denticles in reversing the flows has been described by Du Clos et al., who explained the downward backflow between the denticles and the vortex above the denticles were associated with passive bristling by a high-speed camera. ${ }^{50}$ Some adverse factors, such as backflow, can be inhibited by the passive bristling of shark denticles. The study suggested that shark skin denticles may absorb the negative energy of backflow by passive bristling and delay the onset of turbulence in the boundary layer. In general, the lateral riblets produced by shark skin trap small-scale vortices generated by large vortices between them, thereby forming a local slip on the surface, and the vortices generated on the denticles allow the turbulent boundary layer to reduce the possibility of separation and minimize the pressure drag. However, in fact, the mechanism by which shark denticles reduce drag is not well understood, similar to the riblets of shark. Dynamic or static perspective on drag reduction can have a new discovery, such as in dolphin skin folds and compliance in water with sharks, as widely discussed.

\subsection{Dolphin-inspired drag reduction}

The skins of sharks and dolphins are divided into two extremes in drag reduction and hydrodynamic research. Dolphins' skin are different from the roughness of sharks due to their softness and smoothness. However, dolphins and sharks have something in common in their adaptation and drag reduction to fluid environments. ${ }^{92}$ They are superb predators and their skin is well known for drag reduction. Initially, Gray simulated the drag required and the power requirement for a swimming dolphin and concluded that the power requirement generated by the animal was insufficient to overcome the drag. ${ }^{93}$ 'Gray's paradox', which is well known and explained by Gray, dolphins must maintain a laminar boundary layer to reduce surface frictional drag. ${ }^{93}$ The well-known 'Gray's paradox' is the first result of poor statistics on the swimming ability of underwater creatures. But this paradox, like a treasure, has attracted many researchers to explain the drag reduction of dolphins.

Regarding the mechanism of dolphin drag reduction, research shows that the surface of a dolphin's skin is as flexible as the surface of a shark. Also, the viscoelastic properties of the surface can result in a compliant surface that can absorb energy from the flow and delay transition from laminar to the turbulent boundary layer. ${ }^{38,94-96}$ Also, maintaining the turbulent flow of the boundary layer across the body of the dolphin is the key to
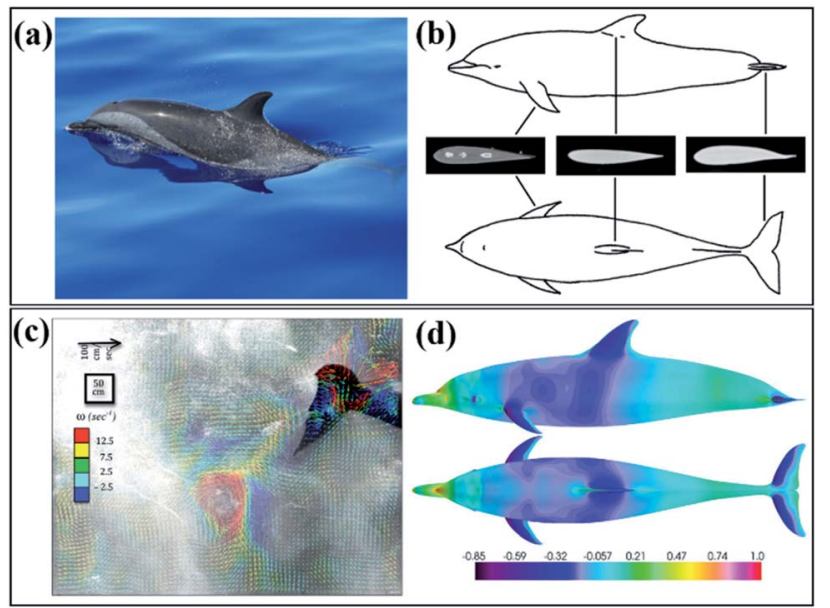

Fig. 6 (a) Bottlenose dolphin (Tursiops truncatus) swimming on the water. (b) Flipper, dorsal fin, and flukes of dolphin streamlined design. Reproduced from ref. 99 with permission from Elsevier, copyright 2019. (c) Distribution of pressure coefficient (flow velocities $U_{\mathrm{e}}=$ $10 \mathrm{~m} \mathrm{~s}^{-1}$ and the turbulence intensity is $\left.1 \%\right)$. Reproduced from ref. 100 with permission from Springer, copyright 2013. (d) Visualization of vortices created by swimming dolphins. Reproduced from ref. 54 with permission from Elsevier, copyright 2014. 
reducing the drag, while the smooth and compliant skin of the dolphin is to maintain the turbulent boundary layer without separating. ${ }^{97,98}$ The paradox about dolphins is gradually explained in terms of dolphins' skin drag reduction. Dolphin drag-reduction mechanisms increase the efficiency of movement and the underwater creatures require less power.

The propulsion generated by the dolphin's movement is also utilized to explain the paradox. Dolphins are found to swim faster than 11 meters per second. To measure the thrust generated by large dolphins, the wake of the dolphins was visualized by using the DPIV (digital particle image velocimetry) method.$^{54}$ Unlike the traditional PIV method, bubbles are used as tracking particles for DPIV (Fig. 6(d)). The measurement results show that the dolphin produces $700 \mathrm{~N}$ of driving force during small amplitude swimming and $1468 \mathrm{~N}$ of driving force during large amplitude starts. Thus, the dolphins' explosive power contradicts the paradox. For actively swimming dolphins, rapid movement causes the boundary layer of the whole body to become thinner; thus, the frictional drag increases and during periodic violent swings, it will deviate from its streamlined shape to produce pressure drag. In fact, the behavioral patterns of diving mammals and birds confirm that energy can be saved by long-term periodic gliding and dolphins have good physical adaptability to breath-hold diving. ${ }^{59,101}$ The drag reduced by moving through the air is greater than the energy cost of jumping. Thereby, dolphins have superb locomotion strategies to conserve their energy (Fig. 7). In addition, morphological, hydrodynamic, and kinematic changes in pregnant dolphins were tested by Noren, ${ }^{55}$ who pointed out that the change in the body shape has a greater impact on the swimming speed of the dolphins and the side shows the excellent hydrodynamic advantage of the streamlined body shape of the dolphins (Fig. 6(c)).

Either the effective drag reduction performance on the surface or the exquisite form of movement is utilizsed to explain the paradox. The basic premise of the focus and reason for

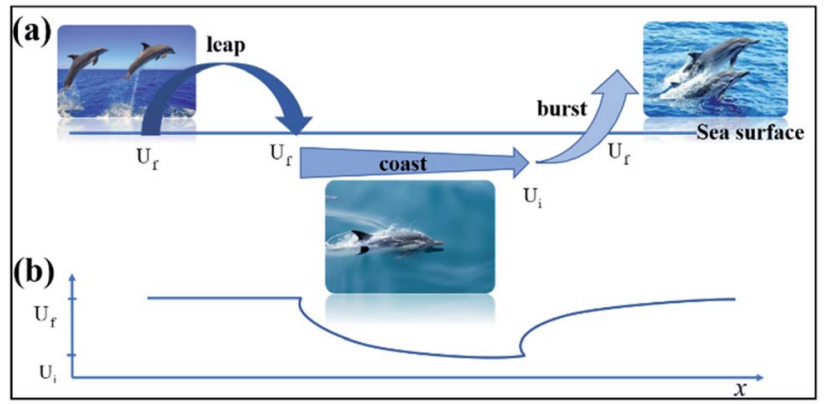

Fig. 7 (a) Schematic description of the swimming process of dolphins Leap, burst, and coast as a fast dolphin sailing a loop. Reproduced from ref. 101 with permission from Oxford University Press, copyright 2002. (b) The speed diagram corresponding to the above picture. The dolphin leaps out of the water at a speed of $U_{f}$. When hopping into the water, the speed maintained is also $U_{f}$, to a shallow depth, and it begins to coast horizontally and gradually slows down until it reaches $U_{i}$, and then rapidly bursts to $U_{f}$ to start the next round of leaping. Reproduced from ref. 101 with permission from Oxford University Press, copyright 2002. dolphin hydrodynamic research is that dolphins maintain laminar boundary conditions. ${ }^{57,93,98}$ However, numerical simulations have concluded that the rigid surface of dolphins is mostly covered by the turbulent boundary layer and only a small part by the laminar flow. ${ }^{\mathbf{1 0 0}}$ It seems that the role of dolphins' compliant skin is to maintain laminar flow and delay transition. In general, the boundary layer has been discussed and analyzed in drag reduction. Using the method of the boundary layer for anti-drag applications can be done using riblets, boundary layer heating, compliant surfaces, polymer additives, and boundary layer acceleration. ${ }^{57}$ Also, part of the mechanisms has been demonstrated in the dolphins' skin.

2.2.1. Dermal ridges and skin folds. Although dolphin skin with shark surface is generally considered to have a good drag reduction effect, the surface of the dolphin is generally described as surprisingly smooth (average roughness $=5.3 \mu \mathrm{m}$ ), as demonstrated experimentally by Wainwright et al., who utilized gel-based profilometry to test a variety of odontocete skins (Fig. 8(b)). ${ }^{62}$ It seems that the dolphins' surface does not affect the boundary layer at the cruising speed. However, observing the slices of dolphin skin, the papillae and ridges of dermal crests were found on many dolphins. ${ }^{\mathbf{1 0 2}}$ The dermal papillae were considered to be the ones that can feel the pressure of the water flow, which is because the liquid in the subcutaneous tissue filled with liquid can flow out or flow into the thin tube, which embeds in the subcutaneous tissue with the change in pressure; thus, it causes the skin to contract or swell up and down, resulting in vibration. Dolphin skin is also thought to respond appropriately to changes in the surrounding turbulent pressure and to act as a buffer against such unequal pressures. The ability to propagate the pressure changes of turbulence on the skin and to wave along with it thereby convert turbulent flow into a more stable laminar flow so as to reduce the frictional drag of water.

The hydrodynamic benefits of riblets parallel to the water flow that has appeared on the surfaces such as sharkskin have been well proven. Riblet-like structures have also been found in dolphins and dermal ridges can be visibly seen when a dolphin swims at high speed.,102 The direction of ridge alignment is crucial for considerations of drag reduction. ${ }^{57}$ Dolphin grooves and shark grooves are arranged in different directions. The sinusoidal grooves formed by dolphin skin folds are perpendicular or horizontal to the water flow on the body. Theoretically, it is believed that the lateral groove induces the generation of secondary vortices, turning sliding friction into rolling friction, and the potential mechanism of reducing frictional drag. ${ }^{92}$ Furthermore, the water flow forms a sliding condition on the surface and induces turbulence augmentation in the boundary layer. Therefore, flow separation is correspondingly reduced and so is the potential mechanism of reducing the pressure difference drag. ${ }^{3}$

2.2.2. Compliant surfaces. Transverse grooves caused by skinfolds were embedded in the dolphin's surface, while movable skin folds were observed in fast-swimming dolphins. The skin folds are thought to be the adaptive, flexible deformations of dolphin skin to the pressure changes in the surrounding flow field. It has also been suggested that skin 

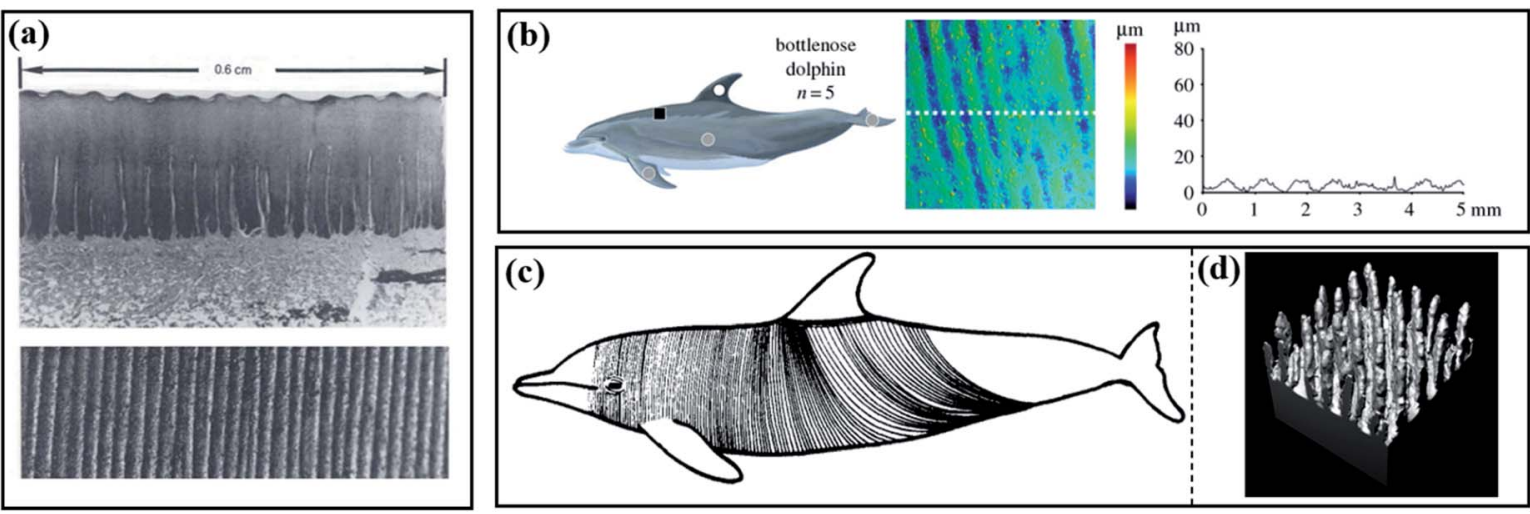

Fig. 8 (a) Dermal ridges and skin folds of a dolphin. Reproduced from ref. 3 with permission from IOP Publishing, copyright 2017. (b) Threedimensional skin structure of a bottlenose dolphin. The surface of the skin is smooth and has no obvious bulges, and the traces of skin furrows and folds can be seen. Reproduced from ref. 62 with permission from the Royal Society, copyright 2019. (c) The distribution of dolphin fold skin throughout the body. Reproduced from ref. 3 with permission from IOP Publishing, copyright 2017. (d) Schematic picture of the internal imitation of a dolphin. Reproduced from ref. 56 with permission from IOP Publishing Ltd, copyright 2006.

wrinkles are the result of active muscle control in dolphins. Then, it gradually developed into active boundary layer control for drag reduction. Carpenter has explained that the active control of dolphin skin is convincing evidence that dolphins possess laminar-flow capability. ${ }^{60}$ The reason for the drag reduction of active boundary layer control is to modify the boundary conditions of the surface to suppress the dynamic significant coherent structure existing in the wall area. ${ }^{104}$ Lorang et al. described that the reason for its drag reduction is to actively move the high shear rate region of the wall into the boundary layer and prevent the flow direction vortex sweeping event to the boundary layer and the upward vortex uplift in the boundary layer. ${ }^{104}$ To further reveal the mechanics of the flexible surface driven by the wall shear stress, the turbulent channel flow at medium and low Reynolds numbers is numerically simulated. The experiment reveals that the passive flow to the wall has at least $3.7 \%$ drag reduction effect and the welldesigned flexible wall can pass the active control to achieve better drag reduction, ${ }^{103}$ and the inhibition of turbulence is considered as a mechanism for skin folds. ${ }^{105}$ Furthermore, inspired by the periodic oscillation of dolphins in the surrounding waters, drag reduction is controlled by the transversal travelling wave. ${ }^{10,106}$

However, active control has been discussed in the context of swimming energy. The simulation found that the wave motion of swimming animals increased the frictional drag of their skin by $20 \%$, the number of which is closely related to the geometry and movement. ${ }^{58}$ Whether active control is an effective dragreduction mechanism has become a controversy. Extensive research has been carried out on passively compliant skin that does not require energy input to the drag reduction system. The artificial skin is replicated based on the structure of the dolphin's epidermis and dermis and the skin's passive viscoelastic properties, which has an excellent drag-reduction effect. ${ }^{98}$ The virtual model was used to simulate the flow around the dorsal fin of the harbour dolphin by Pavlov, which established the relationship between the surface morphology and local flow parameters can be used for the design of multiplate anisotropic compliant walls (Fig. 9(b)). ${ }^{56}$ By measuring the viscoelastic properties of the coating, the effective range of the coating and the flow parameters are predicted and given, and the experimental results obtained are in good agreement with the case of broadband interaction at the low-speed compliance peak. ${ }^{61}$ Also, delaying the transition from laminar flow to turbulence is thought to be the result by which flexible skin reduces drag. The design of drag-reducing compliant coatings inspired by dolphins has been applied to the ship hulls. Also, combining the experimental and numerical techniques gives a drag reduction by almost $3 \%$ in the boundary layer. ${ }^{37}$ Consequently, any realistic modelling effort should consider the different coupled mechanism problems.

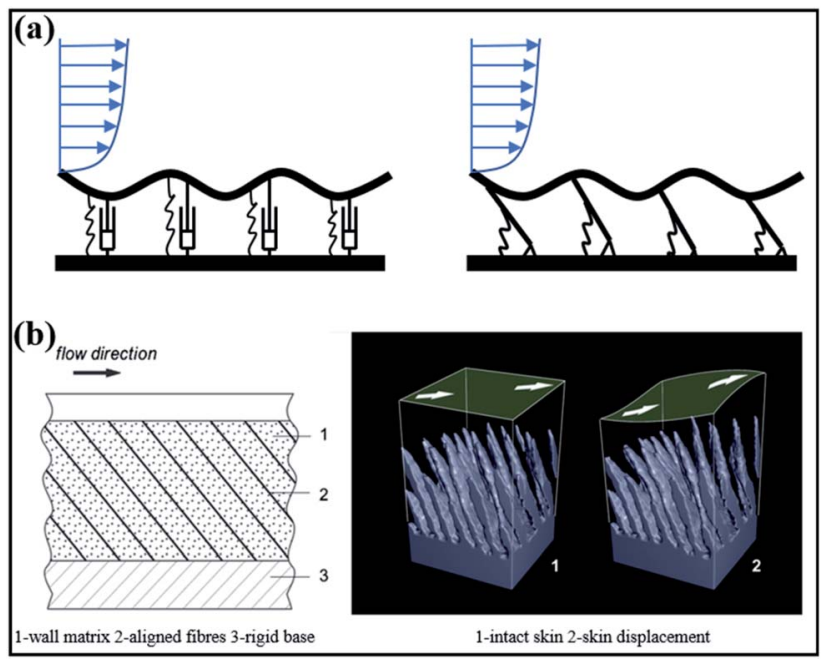

Fig. 9 (a) Compliant wall models. Reproduced from ref. 103 with permission from Cambridge University Press, copyright 2019. (b) Compliant wall structure and the simulation for the deformation of dolphin skin along with shear stress. Reproduced from ref. 56 with permission from IOP Publishing Ltd, copyright 2006. 
2.2.3. Boundary layer heating. The black back and white belly are the most obvious characteristics of the dolphin's skin color. Furthermore, during the dolphin cruise, it is parked near the water for a long time and is exposed to direct sunlight. Therefore, there is a temperature difference of about $9^{\circ} \mathrm{C}$ between the skin of the dolphin and the boundary layer covering the skin. ${ }^{109}$ From a fluid dynamics perspective, it is recognized that the increase in temperature causes the viscosity coefficient of the liquid to decrease and the fluidity to increase, which is due to the reduction in cohesion between the molecules. Also, the temperature may also affect the boundary layer, such as delaying the transitions and preventing vortex shedding. F. C. Li et al. suggested that temperature affects the Reynolds number shear stress near the boundary layer. ${ }^{110}$ By adjusting the temperature of the boundary layer, the viscosity of the area within the boundary layer is reduced, the velocity distribution of the boundary layer is changed, and the boundary layer is kept stable.

According to the boundary heating drag-reduction mechanism, a graphene composite material that simulates the elasticity and thermal conductivity of the dolphin skin is prepared by L. Tian et al., which found that the contact area between the material and the water beads did not increase significantly and the dynamic viscosity decreased, which were the reasons for drag reduction. ${ }^{109}$ Only when the fluid is heated instantly on the surface of the object, the reduction in the dynamic viscosity of the fluid will lead to the reduction in the drag. The simulation result of Peeters et al. has shown that instantaneous density and dynamic viscosity fluctuation are the reasons for drag reduction of the turbulent boundary layer. ${ }^{\mathbf{1 1 1}}$ The dolphin with characteristic black back and white belly and aquatic routes have been considered by Hassanalian. ${ }^{\mathbf{1 0 8}}$ For the effect of temperature on the surface resistance of the aquatic organisms, a flat plate with

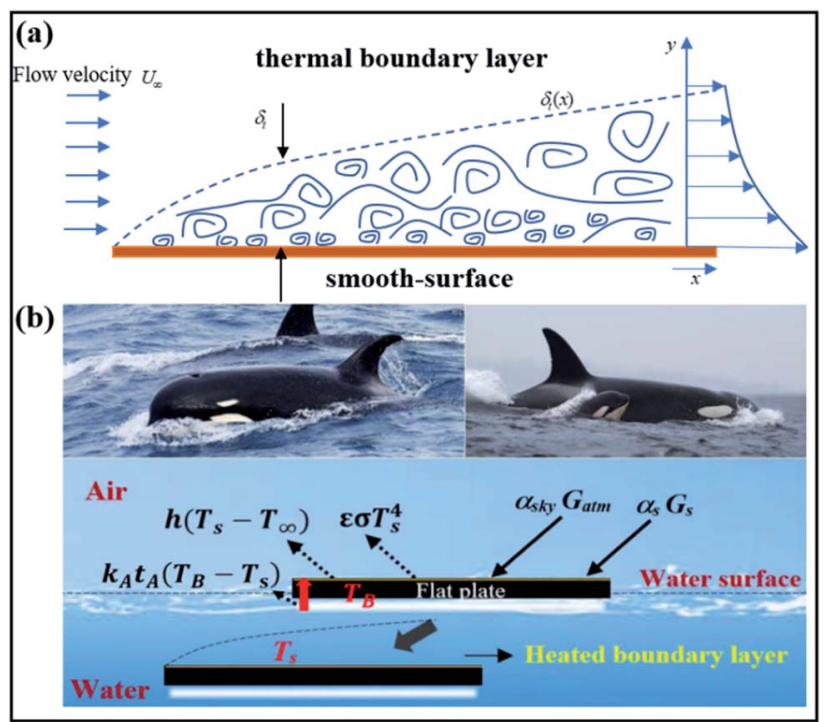

Fig. 10 (a) Schematic diagram of the thermal boundary layer over the wing. Reproduced from ref. 107 with permission from Elsevier Ltd, copyright 2017. (b) Schematic diagram of energy conservation in the heated boundary layer. Reproduced from ref. 108 with permission from Elsevier Ltd, copyright 2019. black and white color was used to simulate drag reduction on the dolphin surfaces. The results show that the temperature effect boundary layer is very effective for drag reduction and dolphins reduce the frictional drag of their skin by $7 \%$ (Fig. 10). The drag-reduction mechanism of boundary-layer heating is also reflected in migrating birds (albatross). ${ }^{107}$ This mechanism will improve the design by applying inspirational colors that can help the aircraft and vehicle to increase the endurance. Direct sunlight on the skin surface heats the boundary layer, which causes the dynamic viscosity and density of the water medium to change to reduce the drag. Consequently, the fish's color seems to play an important role in drag reduction. Shark or dolphin flexible skin of realistic modelling effort should consider a static move from dynamic and active to passive.

\subsection{Other aquatic animals-inspired drag reduction}

The species of other aquatic families display a variety of shapes and sizes in nature. Aquatic animals have similar hydrodynamic characteristics due to their similar living environment. The superb sports strategy and fluid properties of aquatic animals in water are amazing. Reynolds number (Re) is the expression of the ratio of inertial force and viscous force, which has been supposed to describe the surrounding flow field information of aquatic animals of different sizes. When aquatic animals are in motion, the surrounding flow field is mostly turbulent and the Reynolds number is about $5 \times 10^{5}$ to $5 \times 10^{6}$. At such a high Reynolds number, the inertial force is more important, and the movement pattern and streamline are the reasons behind the aquatic animals to maintain low drag.

Regardless of the active movement or passive use of posture and internal compliance of the form and fins, ${ }^{\mathbf{4 4}}$ it is the basic ability of aquatic animals to maintain the stability in complex three-dimensional turbulent waters. Flow adaptation and vortex capture are considered to be the main mechanisms of aquatic animals' drag reduction in water. ${ }^{\mathbf{1 1 2}}$ However, fish are widely discussed as the most abundant aquatic animal. For instance, the fish behind the cylinder will remain stationary in a unique posture and movement form, which is known as Kármán gait. ${ }^{12}$ Also, in contrast to the need to overcome turbulent flow, the aquatic animals are able to extract energy from the vortices created by the environment to maintain downstream flow. ${ }^{\mathbf{1 3 , 4 4}}$ Novati et al. conducted the experimental analysis of two selfpropelled fish and found that the followers in the wake area used reinforcement learning to adapt to the leader's swimming kinematics in order to maintain a synchronized tandem manner. These motion patterns can reduce the follower's energy consumption by $30 \%$ and improve the swimming efficiency by $20 \% .^{113}$ The oxygen consumption of the fish during exercise was measured by; ${ }^{8}$ it was a surprise to find that fish consume less oxygen at high turbulence than at low turbulence at medium and high swimming speeds. The explanation for this is that it is not easy to think of fluid adaptation (Kármán gaiting) as a specific behavior in aquatic animals motion but rather to harness high turbulence energy that changes with time. ${ }^{8}$

The streamlined body optimizes the pressure gradient of the entire body so that the fluid body squeezes the back, abdomen, 
or side of the aquatic animals to a minimum, and also reduces the effect of differential pressure drag when swimming at high speed. ${ }^{11}$ The streamline has a rounded front and pointed rear, smooth surface, and slightly droplet shape; thus, good pressure gradient and laminar flow in water are regarded as the characteristics of a streamlined structure. For example, it is rare to see large undulations and sharp edges and corners on dolphins and sharks but smooth and regular surfaces. At the widest point of the fish, turbulence is induced, thus delaying the separation of the boundary layer. Blob et al. has a strong interest in the drag reduction of Hawaiian stream fish in the environment of climbing turbulent waterfalls. For the comparison of different characteristics of river fish, experimental research has found that lower head heights are connected with contact to high-flow regimes. ${ }^{35}$ The fluid flows backwards along with the streamlined head and the fluid in the boundary layer will gradually slow down and pressurize. If the flow is under pressure gradient, it will further decelerate. Finally, the kinetic energy of the fluid in the entire boundary layer is not enough to maintain the flow for a long time and cause separation. Studies have shown that fish are widely discussed as the most abundant aquatic animals' surface, which are covered with a non-smooth surface to delay separation, such as the grooved dolphin surface. ${ }^{21}$

On the other hand, the destruction of the streamlined structure of the aquatic animals will lead to an increase in the drag. ${ }^{55}$ Quicazan-Rubio et al. tested the negative impact of pregnancy physiology on fish. ${ }^{\mathbf{1 1 4}}$ In the hydrodynamic experiment, the maximum volume increase caused by pregnancy physiology was about 43\% (Fig. 11(a)). Also, the expansion of the abdomen during pregnancy leads to the expansion of the separation area of the tail and the tightening of the muscles, which makes the fish lose its flexibility; thus, the drag and flexibility of the fish are reduced.

2.3.1. Morphology. Aquatic animals seek protection while living in water and thus have evolved different types of scales. The scales of other aquatic animals appear in different shapes and sizes. Some fishes have shown advantages in drag reduction compared with other aquatic animals. Nearly $62 \%$ of cartilaginous fish scales are defined as drag-reducing scales, of which $25 \%$ are identified as wear-resistant scales. ${ }^{32}$ Therefore, fish with scales, as a representative of aquatic animals, are described to discuss the drag reduction mechanism.

The scales covered on the surface of the fish show a wide range of morphological diversity, which has also been found to have drag-reduction effect. The scales on most fish are cycloid and comb-shaped scales; the main difference is that there are several small comb-shaped spines on the tail end of the comb scales. ${ }^{78}$ The lateral line, as a sensory organ unique to fish and aquatic amphibians, is mainly distributed on the cycloid scales on both sides of the fish body. It is a result of a high degree of differentiation in the skin sensory organs, which can feel the function of water flow and the function of identifying and locating obstacles. Liao indicated that the lateral line of the body of the rainbow trout has a greater influence than the visual body kinematics in the vortexes street. ${ }^{1}$ It can be explained that the shoal of fish can feel a change in the water pressure and show a positive response.

Unlike the cycloid, the fish body can be covered with a large number of comb-shaped scales arranged regularly. The scales of bony fish (Ctenopharyngodon idellus) were analyzed through numerical simulations, which was thought to produce the
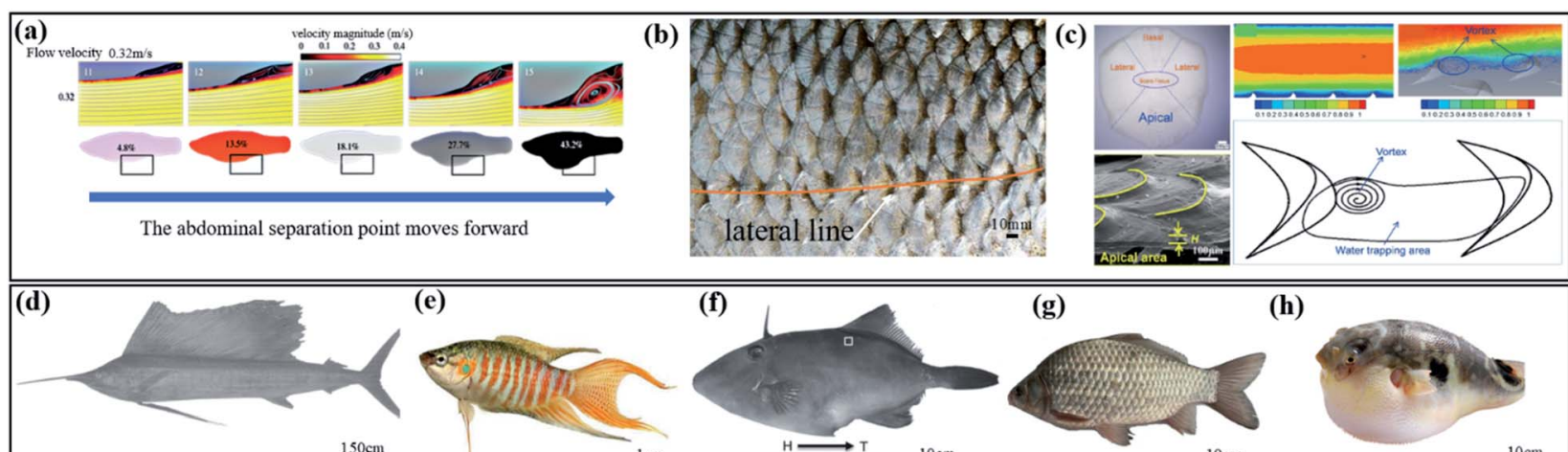

(e)

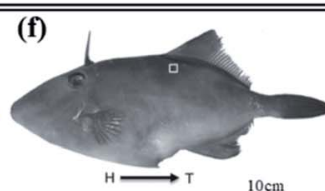

(g)

(h)
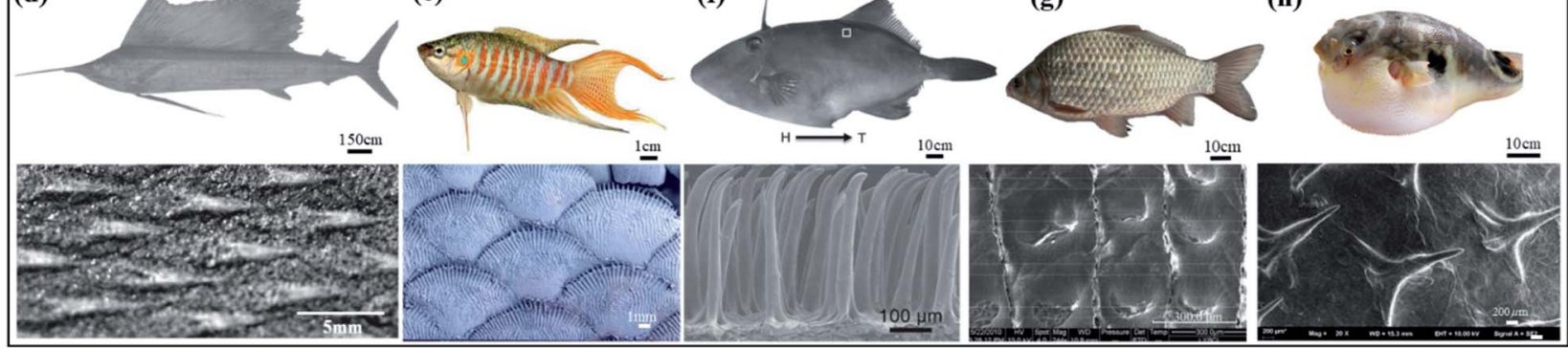

Fig. 11 (a) The effect of the change in the pregnant fish body shape on the surrounding flow field. Reproduced from ref. 114 with permission from the Royal Society, copyright 2019. The morphological changes in the fish bottom cause the boundary layer to separate earlier due to pregnancy, thereby increasing the drag. (b) Cycloid scales and lateral line of fish (Rutilus rutilus). Reproduced from ref. 78 with permission from Cambridge University Press, copyright 2019. (c) Water-trapping effect of fish scales (Ctenopharyngodon idellus). Reproduced from ref. 41 with permission from Springer Verlag, copyright 2017. (d) The skin of sailfish. Reproduced from ref. 30 with permission from AIP Publishing, copyright 2008. (e) Scales of a paradise fish (Macropodus opercularis). Many bulges at the end of the scales. Reproduced from ref. 78 with permission from Cambridge University Press, copyright 2019. (f) The skin of filefish ( $\mathrm{N}$. septentrionalis). Reproduced from ref. 24 with permission from Wiley-VCH Verlag, copyright 2014. (g) The skin of fish (Carassius auratus). Reproduced from ref. 115 with permission from Science Press, copyright 2012. (h) Spines of the adult puffer. Reproduced from ref. 116 with permission from Wiley-Liss Inc., copyright 2020. 
"water-trapping" effect and to form surface synovial lubrication $^{40}$ with frictional drag, which decreases by $3.014 \%$ when $0.66 \mathrm{~m} \mathrm{~s}^{-1}$ flow rate is tested. ${ }^{41}$ Staggered fish scales such as grooves may be associated with drag reduction, as demonstrated experimentally by Muthuramalingam et al., used the fish scales pattern (European sea bass) to simulate and compared with the result of the surface oil-flow experiment. ${ }^{39}$ It seems that high-speed and low-speed stripes are staggered on the fish scale, and low-speed stripes are mainly distributed in the central area of the scale, while high-speed stripes are distributed in the overlapping area of the scale. Inspired by the water film effect on the scales of the crocodile's abdomen, the hexagonal drag reduction marine board is replicated on the macrostructure and the drag reduction effect is best at $6.3 \%$ when the speed is $0.025 \mathrm{~m} \mathrm{~s}^{-1} .^{42}$

In recent years, pufferfish have been extensively studied. Studying the paradox of boxfish swimming is not due to the bulky size of the boxfish that leads to increased flexibility. On the contrary, the posture changes the surrounding flow field and increases the flexibility. ${ }^{31}$ To further reveal the effect and flexibility of studying the drag reduction of puffer, Zhou, Cui, et al. established the 3D curved surface structure of pufferfish and expanded their surface into planes for analysis. ${ }^{\mathbf{1 1 7}}$ Inspired by the body spines of the pufferfish, ${ }^{118,119}$ the simulation shows that the drag reduction rate of the non-smooth surface imitating the body spur of the puffer is $12.94 \%$ when the water velocity is $5 \mathrm{~m} \mathrm{~s}^{-1}$. $^{116}$

However, the scales of some fish are also considered to have no drag reduction. There are many $\mathrm{V}$-shaped protrusions on the skin of the fast-moving swordfish pointed downstream by Sagong et $a l^{30}$ It is assumed that the $\mathrm{V}$-shaped protrusion structure does not have the function of drag reduction under the simulation experiment. In addition, the filefish have a rough bony skin described by Cai et al. ${ }^{24}$ which has anisotropic oil repellency on moist surfaces and explained that the oleophobic properties are from head to tail but show lipophilicity from tail to head. Fish skin with scales has a rich texture to achieve the best drag reduction effect but the mucus attached to the fish surface seems to have a coupled drag-reduction mechanism with the texture.

2.3.2. Mucus. The ever-changing scales on the surface of aquatic animals were found to have drag reduction and other practical functions. Also, mucus has a non-negligible effect on fish drag reduction. Fish scales are covered with a thin layer of mucus secreted by the fish mucus cells. According to the experiments of Rosen and Cornford, ${ }^{\mathbf{1 2 2}}$ mucus gives fish a smooth feeling and has a good drag reduction performance (drag reduction of $65.9 \%$ when the mucus concentration is $5 \%$ ). A variety of fish mucus were tested in aqueous solutions with different dilutions using a flow rheometer. ${ }^{122}$ It seems that drag reduction of the mucus effect from the predator (Pacific barracuda) is better. Polymer additives that are inspired from mucus such as small amounts of high molecular weight polymers in water, ${ }^{123}$ micellar surfactant systems, ${ }^{79}$ bubbles, ${ }^{\mathbf{1 2 4 - 1 2 6}}$ coatings, ${ }^{29,127}$ and even solid spheres ${ }^{\mathbf{1 2 8}}$ can reduce drag, which has important potential implications for reducing energy losses in pipelines and underwater vehicles. ${ }^{17,18,129}$

In recent years, riblets have been extensively studied, and fish mucus and riblets of fish are considered to have a coupling effect on drag reduction, thus affecting the structure of the flow field around the fish. ${ }^{130}$ The reason for the drag reduction of fish mucus is often explained by slip theory. Also, the mucus is sheared and stretched evenly in the near-wall region, which leads to the stretching of the near-wall stripes and the reduction of the strength of the quasi-flow vortex structure of the buffer layer. The weakening of these vortices causes a reduction in the momentum exchange of the high/low bands. The DSD/SST method is utilized to simulate fish (two-dimensional two-layer fluid model) wrapped in mucus swimming in a non-Newtonian fluid. The results show that fish mucus is a low-shear liquid with drag reduction, the

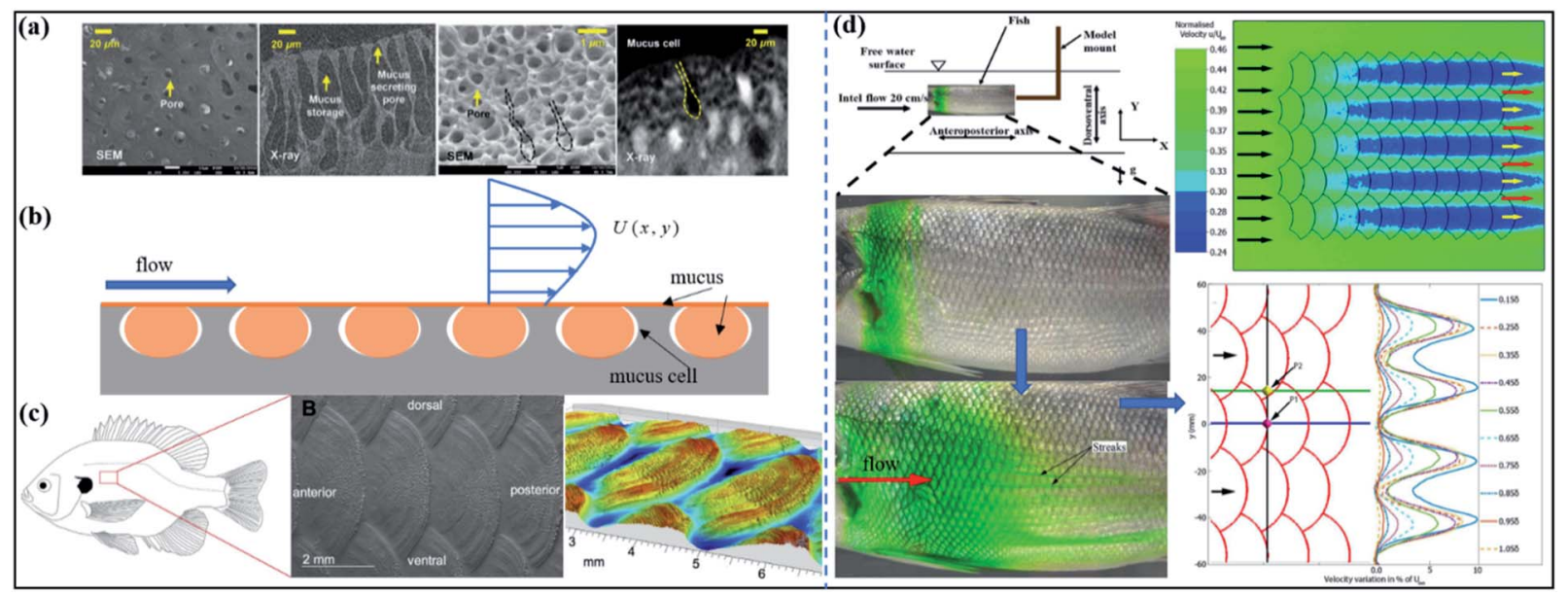

Fig. 12 (a) SEM and X-ray images of the morphological structure of mucus storage. Two pictures of loach on the left, two pictures of hagfish on the right. Reproduced from ref. 120 with permission from Royal Society of Chemistry, copyright 2019. (b) Schematic diagram of bionic surface slippage injected with mucus. (c) Scales from the central region of fish (Lepomis macrochirus). Reproduced from ref. 121 with permission from Elsevier $\mathrm{GmbH}$, copyright 2016. (d) Fish scales and mucus work together to form streaks. Reproduced from ref. 39 with permission from Company of Biologists Ltd, copyright 2019. 
effect of damping, and increasing propulsion. ${ }^{4}$ Inspired by the mucus secretion and storage system, Lee et al. explored a lubricating oil injection surface to increase the sustainability of the injected lubricant. ${ }^{\mathbf{1 2 0}}$ It still maintains excellent drag-reduction capacity under high shear flow experiments and its drag reduction rate reaches about $18 \%$, which provides a design idea that can maintain stable battery life while maintaining a high dragreduction effect (Fig. 12(b)). As shown in Fig. 12(d), after the experiment, the distribution of the pigment on the fish can be obtained by alternating the low-speed and high-speed streaks. The boundary layer of the fluid under the action of scales and mucus appears to sink and converge in steps, such as the converging vortex and diversion in a riblet but it is different from the riblet in the scale. ${ }^{39}$

In addition to the hydrodynamic function, the mucus secreted by fish mucus cells also has the function of immunity, thus preventing the invasion of foreign bodies and pathogens, and maintaining the osmotic pressure of the body. ${ }^{\mathbf{1 3 1}}$ Therefore, mucus is a highly multifunctional substance and has been the focus of research in recent years. At the same time, the viscosity also has a high drag reduction rate, leading to a fish surface that not only exhibits scale changes in the flow field structure but the drag reduction is also affected by the mucus. Whether or not mucus plays an important role in flow separation control has not been explained. Still, the mixing of mucus and water changes the density and viscosity of the flow medium in the boundary layer.

\section{Fabrication technology}

The inspiring natural surfaces at the micro-scale can be observed by the microscope and have functions and characteristics related to fluid mechanics. Various fabrication methods used in drag reduction are shown in Table 2. The creation of a micro-scale drag-reducing surface becomes the key to breakthroughs in drag-reduction technology. To study the hydrodynamic effect of the surface structure of the fish scales and to apply it in practice, in this section, therefore, the manufacturing methods and functions of biologically-inspired drag-reducing surfaces will be briefly introduced in recent years.

\subsection{Direct micro-imprinting method}

Direct bio-replicated micro-imprinting technology directly takes the real skin as the object, like a mirror, and mirrors the real surface to the maximum. The accuracy of direct bio-replicated micro-imprinting is higher than other direct bio-replicated methods. Y. Luo and Zhang explored a new shark skin replication accuracy by using direct bio-replicated micro-imprinting technology. ${ }^{132}$ After being tested on the accuracy of imitation shark skin, it is found that the accuracy of reproduction and the degree of reduction is high. Micro-imprinting technique was also utilized for replicating the biomimetic shark-skin in order to explore the wetting properties of shark skin replicas and the result indicated a duplicated surface to show a better effect on the wettability feature. ${ }^{\mathbf{8 6 , 1 3 3}}$ It seems that using this technique can be improved in their self-cleaning and antifouling properties. Chen et al. proposed the large-scale amplification of biological replication technology to scale up the scale of the shark through the solvent-swelling polymer. ${ }^{134}$ The experimental results show that the shark scales can be amplified by $140 \%$ and guarantee more than 95\% accuracy. The large-scale shrinkage bio-replication technology, which is opposite to the large-scale amplification bio-replication technology, is also beneficial for the application of drag-reduction materials in various environments. Thus, Chen et al. proposed a scalable special direct bio-replication micro-imprinting technology to adjust the optimal drag-reduction area of shark skin. ${ }^{135}$

The UV-curable material is cast on real shark skin and shrinks during the formation of the negative template. The final imitation sample prepared was tested and the shrinking ratio reaches $23 \%$ compared with the real shark scale. Dragreduction experiments found that the drag-reduction effect of the imitation samples is significantly better than that of real skin in high-speed flow. The manufacture of drag-reducing materials and the testing of drag-reducing experiments are the constants in drag-reducing research. This technology was used to synthesize active shark skin, polymer additive grafting (PAG), and micropore diffusion was proposed for the drag reduction test. A comparison of drag-reduction experiments shows that the combined drag-reduction effect of the additives and bionic shark skin is better. ${ }^{169}$ The bio-inspired surface was fabricated by the bio-aid method to explore shark skin hydrodynamics. The subtle difference from direct bio-replicated micro-imprinting is that the longitudinal and transverse was respectively stretched 1.5 times as the primary (Fig. 13(b)). Longitudinally-stretched shark-skin was found to have better hydrophobicity and drag reduction. ${ }^{136}$ Also, T. Kim et al. utilized the manufacturing method of the top-down micro-imprint method to create micro-ridges of the layered metal structure in order to reduce the drag ${ }^{137}$ because the metal surface has wear drag and corrosion drag. The direct bio-replicated microimprinting method is a commonly used method to replicate the biological surfaces, with high replication accuracy and excellent moulding effect. But it cannot be processed in large quantities.

\subsection{Bio-replicated rolling method}

The bionic surface produced by the direct reproduction method is only a small area of moulding. However, a large-area rolling riblets structure method was proposed by Hirt and Thome, which also designed and discussed the feasibility of a new winding concept for the continuous forming of small negativeriblets structure rolls. ${ }^{184}$ In the batch processing of imitation shark surfaces, sometimes additional steps and steps are required to achieve micro-rolling in order to eliminate the effect of scaling of the wedge angle on the back on the surface quality. Y. Luo and Zhang proposed a large-area forming method by using biological shark skin roller rolling. ${ }^{172}$ The two steps of pretreatment and fixation are to clean the shark skin and achieve the stiffness and smoothness of the treatment.

In order to eliminate the effect of scaling of the wedge angle on the back on the surface quality, sputtering and lithography techniques have been used successively. The processed shark 

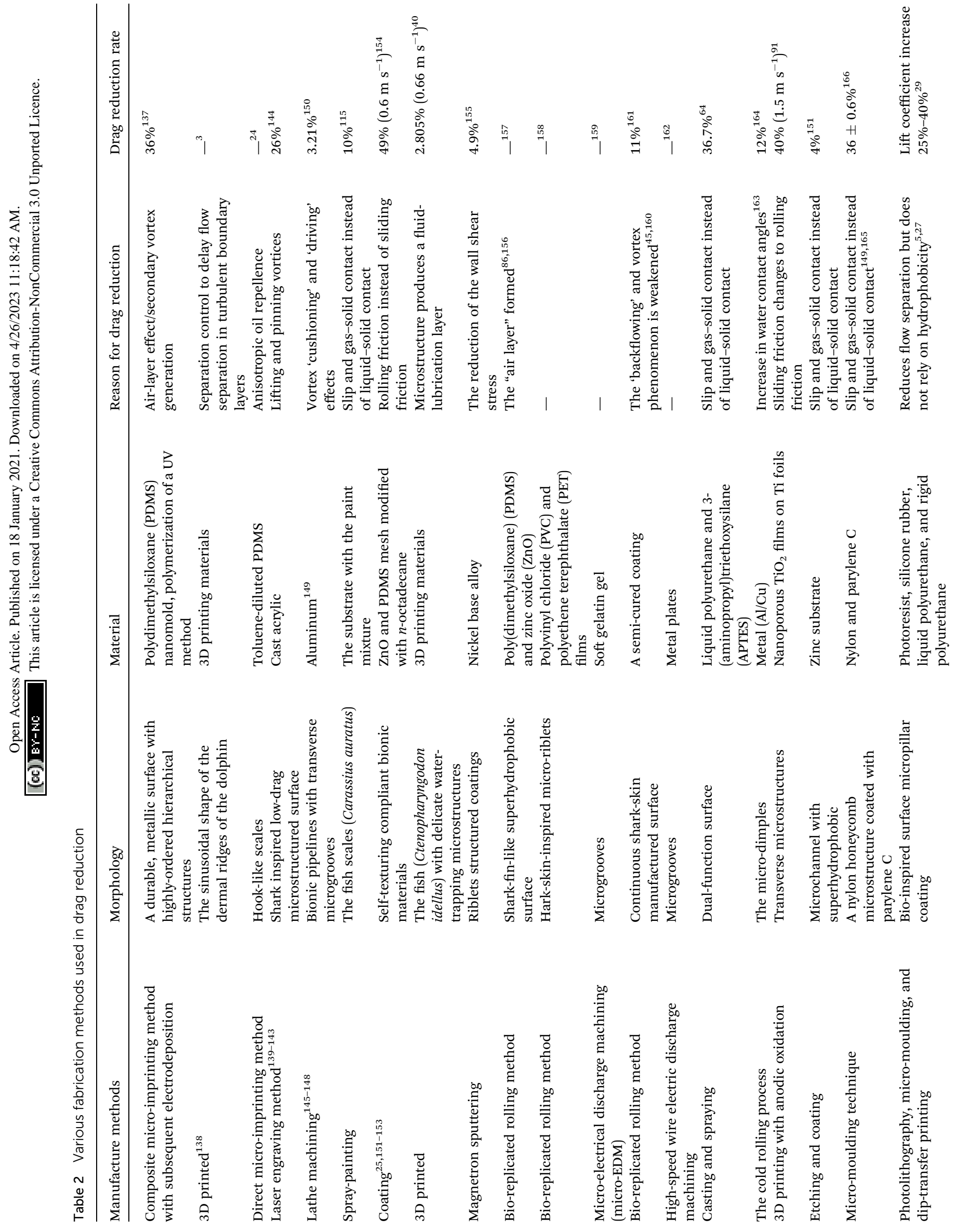


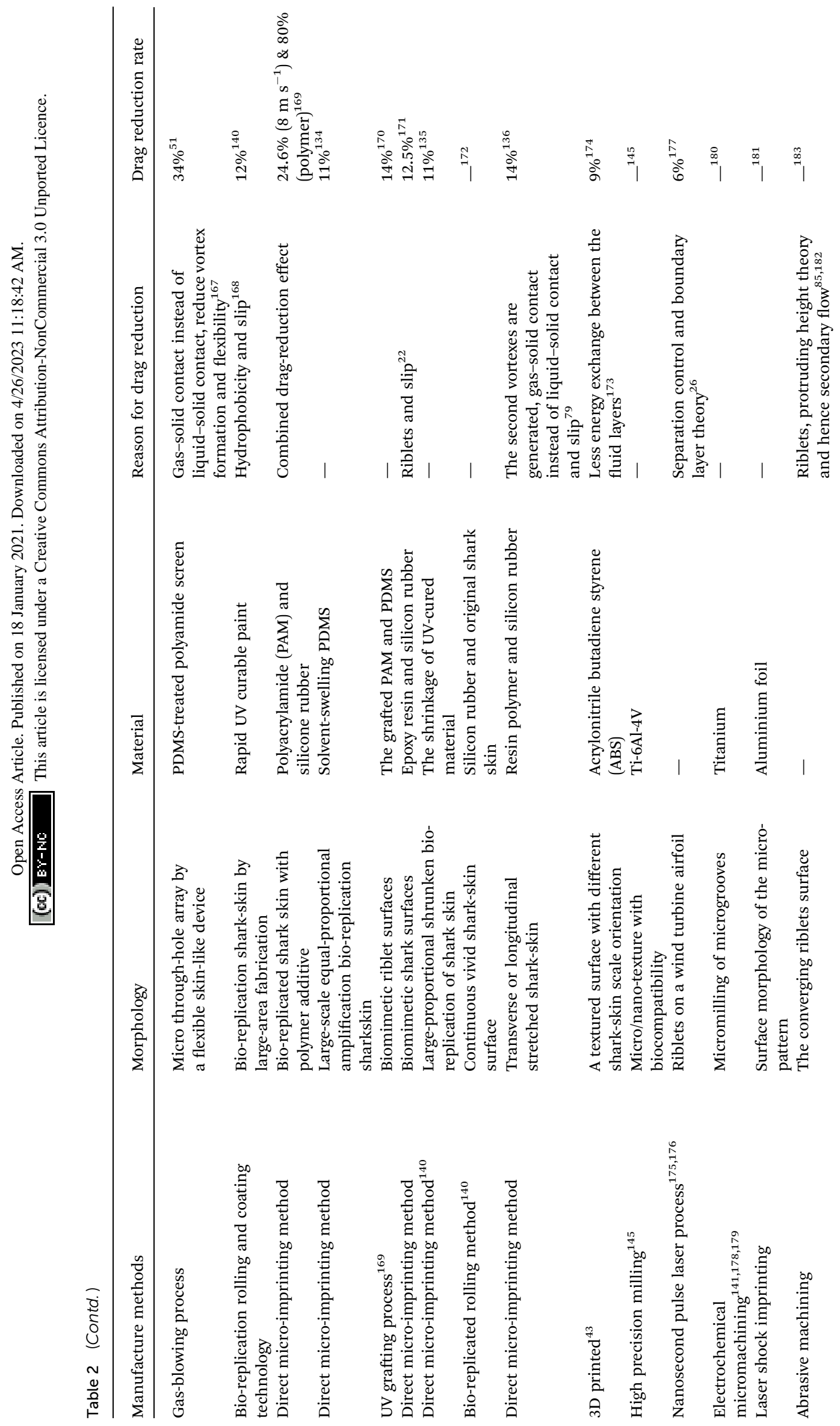




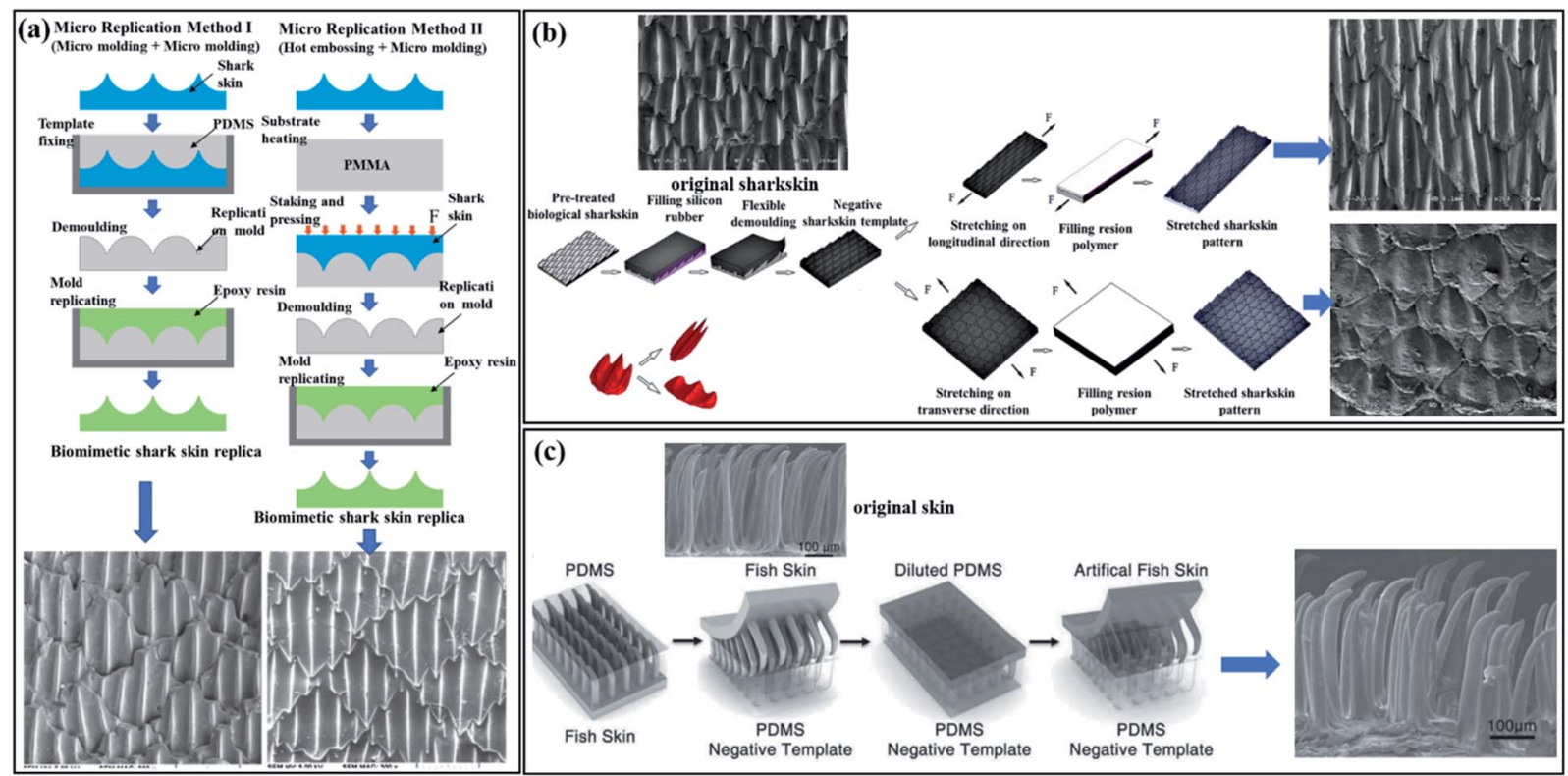

Fig. 13 (a) Brief process of shark's two types of direct bio-replicated micro-imprinting method. Method I: PDMS mixed with the curing agent is utilized in the positive template and then epoxy resin is added as a copy surface material to be used in the positive template. Method II: the hot embossing method for the positive template (the flat polymethyl methacrylate (PMMA) and epoxy resin are the same as method one as the surface material to be utilized in the positive template). The final replicated surface has a high degree of similarity with real skin in performance. Reproduced from ref. 133 with permission from American Scientific Publishers, copyright 2014. (b) Schematic of the improved direct bioreplicated micro-imprinting method. The replicated shark surface is stretched horizontally or vertically to achieve higher drag reduction. Reproduced from ref. 136 with permission from Springer Berlin Heidelberg, copyright 2015. (c) Illustrations of the reproduction of the prototype with a biological skin-like hair. On the far right is the SEM image of the skin replica. The replicated skin maintains a high degree of similarity with the original skin and the oleophobic properties are not lost. Reproduced from ref. 24 with permission from Elsevier GmbH, copyright 2014

skin and the 704-silicon rubber and straps are glued together to form a roller of shark-skin. The final product is prepared by rolling. After testing, the results show that there is almost no formation error in the direction of width and the formation accuracy in the direction of scale height is about $90 \% .{ }^{172}$ Subsequently, the drag reduction experiments in the water tunnel further showed that the imitation surface has a good drag reduction effect and the maximum drag reduction rate reaches $11 \% .{ }^{161} \mathrm{~A}$ large-area roll-formed shark bionic surface based on fast UV-curing paint was proposed by Chen et al. ${ }^{140}$ which was tested and found to have better self-cleaning ability and drag reduction effect than a simple two-dimensional riblet structure. Guo et al. proposed the method of roller embossing to replicate the shark skin-inspired micro-riblets. ${ }^{158}$ The UV-LIGA technique was used on the molds for the manufacture of negative microstructures and the micro-riblets were transferred to the ethylene (PVC, $80{ }^{\circ} \mathrm{C}$ ) and polyethylene terephthalate (PET, $70^{\circ} \mathrm{C}$ ) films by the roller embossing process (Fig. 14(b)). An et al., based on the method of roller imprinting, transferred the pressed shark fin-shaped figure to the surface of the PDMS through soft lithography, and finally, a fin-shaped composite material was obtained by hydrophobic treatment, which effectively realized the directional driving of the droplets and drag reduction performance. ${ }^{157}$ Using wire masks as a rolling cylinder, it can be pressed to form an ellipsoid-shaped microdimples on the polymer surfaces. Simple, fast, and rapid prototyping are considered as the advantages of the modified method and increase the contact angle and have an excellent drag-reduction effect, as reflected in the surface test. ${ }^{164}$ As shown in Fig. 14, by using the bio-replicated rolling method, it is effective to produce a large-area continuous drag reduction surface and truly reproduce the biological surface, which has some advantages including simple fabrication and low cost. ${ }^{157,172}$ However, roll forming surfaces also have low accuracy and long manufacturing cycles. Rapid prototyping 3D printing technology further improves the accuracy.

\subsection{D printing}

3D printing technology is classified as a rapid way of fabrication, which is also known as additive technology and is based on digital model files, using adhesive materials (powdery metal or plastic) to construct entities via layer-by-layer printing. 3D printing technology is widely used in the preparation of biologically-inspired surfaces due to its low cost and the ability to quickly fabricate complex and small three-dimensional entities. A synthetic, flexible shark skin membrane was first designed and fabricated by Wen et al. through the 3D printing technology. ${ }^{43}$ The biomimetic shark skin was composed of flexible lining and shark scales fabricated by 3D technology, which realistically restored the sharkskin flexibility, softness, and surface drag reduction scales. The ability to manufacture synthetic bionic shark skin using 3D printing technology makes it possible to test hydrodynamics of different surface parameters. Lauder et al. explored a small-scale manufacturing 

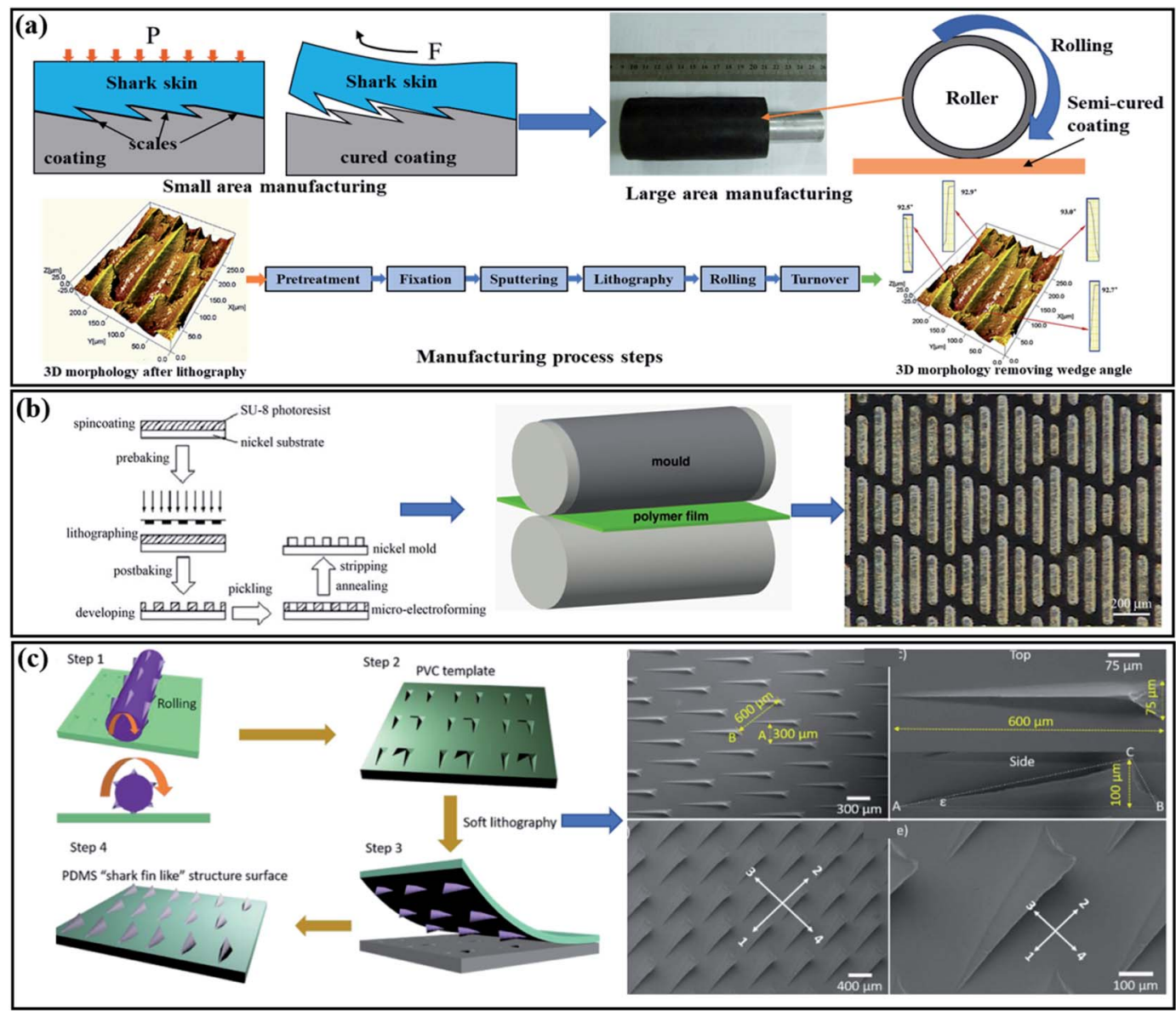

Fig. 14 (a) Image of the bio-replicated rolling method. Compared with the direct bio-replicated micro-imprinting method, large-area manufacturing is its main feature. The real shark skin is processed by pre-treatment and fixation, sputtering, and lithography, which eliminate the disadvantages of the wedge angle and make the shark-skin softer to make it into a roller. Finally, a bionic surface is prepared through the rolling and turnover steps. Reproduced from ref. 172 with permission from Company of Elsevier B.V, copyright 2013. (b) Image of the replication of shark skin-inspired micro-riblets by the rolling method. Imitation of the shark micro-rib structure as the surface of the roller. Reproduced from ref. 158 with permission from Institution of Engineering and Technology, copyright 2017. (c) Bio-replicated rolling method to manufacture a shark-finlike surface. Reproduced from ref. 157 with permission from Elsevier B.V, copyright 2019.

technology for shark scales. ${ }^{2}$ Imitations smaller than the actual shark shield scale were printed using the 3D printing technology and two-photon lithography, as shown in Fig. 15(a).

The flexibility and reproducibility of this technology have excellent application prospects in reducing the drag on biological surfaces. Wen et al.'s follow-up research on the hydrodynamic performance of bionic shark skin through 3D printing technology ${ }^{90}$ shows the flexibility of 3D printing technology, which makes it possible to realize the denticles' graphic arrangement, denticles' shape research, and denticles' size research. ${ }^{63,90}$ There are also experiments using simplified shark riblets. A textured surface with a series of directional scales was designed and manufactured by using the 3D printing technology, and Dai et al. utilized a rheometer to test the best dragreduction effect when the ridge and fluid flow direction are $90^{\circ}$, as shown in Fig. 15(c). ${ }^{174}$ 3D printing technology is the most widely used method in the molding and manufacturing of complex structures. Practicality and versatility are suitable for combining with other methods to prepare biologically- characteristic surfaces. However, the manufacturing accuracy and source model establishment are still points that need to be broken through. The development of imaging technology, such as micro-CT scanning, gel-based surface profilometry, and histology bring new exploration directions for the establishment of bionic models. 3D printing technology can achieve high-precision and realistic reduction of biological surfaces in a proportional proportion, which is not efficient in manufacturing and molding; thus, it is widely used in scientific research to test the characteristics of biological surfaces, such as drag reduction and hydrophobicity. The development of large-area manufacturing technology of microstructures has expanded into high-precision and large-area directions. In the next section, various methods of processing microstructures will be introduced one after the other.

\subsection{Precision machining}

The development of microstructure processing technology has improved the drag-reduction performance of the surface due to 


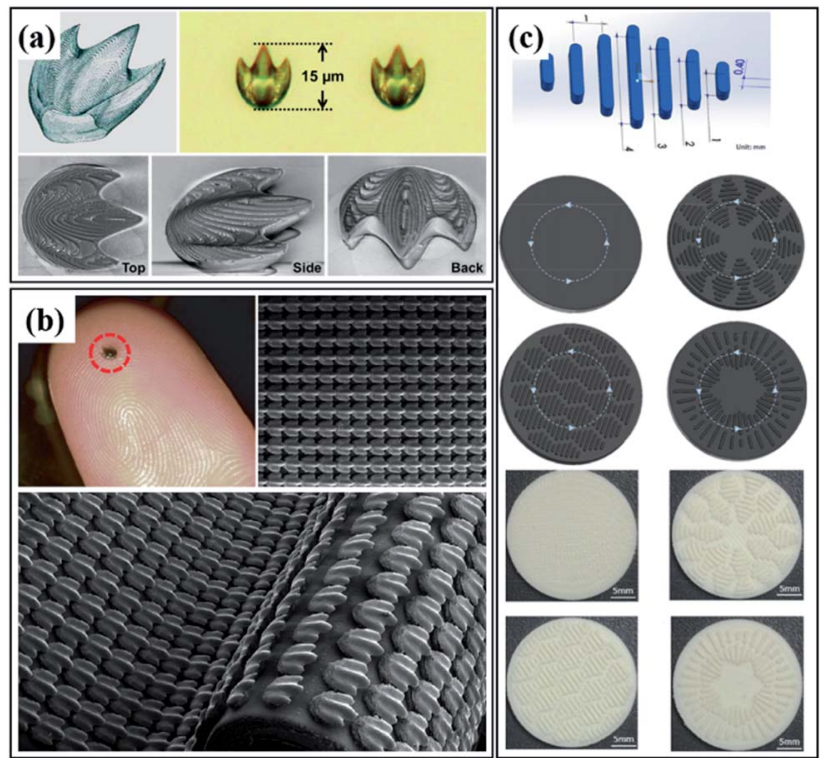

Fig. 15 (a) Images of the 3D print of a mako shark (Isurus oxyrinchus) skin denticle. Reproduced from ref. 2 with permission from Company of American Physical Society, copyright 2016. (b) 3D-printed shark denticles are combined with a flexible substrate to prepare a bionic shark skin (each denticle is about $1.5 \mathrm{~mm}$ length). Reproduced from ref. 43 with permission from Company of Biologists Ltd, copyright 2014. (c) Three-dimensional modelling of the shark skin surface and 3D printing formed by arranging the scales in different directions. Reproduced from ref. 174 with permission from Company of Springer Nature, copyright 2018

the hydrophobicity and 'guide' of the microstructure. Also, significantly increasing the wear resistance of the surface due to the reasonable size can store the lubricating oil. Therefore, bionic microstructures are widely used. Microstructureprocessing technology mainly includes laser etching, microEDM, electro-hydraulic beam processing, self-excited vibration processing method, mask chemical etching method, mask electrochemical processing, and grinding. ${ }^{148}$ The bionic dragreduction surface is based on the microstructure and microstructure manufacturing plays an important role in the dragreduction effect. The precision machining method is a cutting technique that utilizes a small diameter end milling cutter to remove the excess material from the processed workpiece. It is widely used for manufacturing micro-complex parts. ${ }^{146}$ Initially, using a single-crystalline diamond endmill to process the oxygen-free copper surface has obtained extremely smooth, high-precision non-smooth surfaces, such as the micro-pit array, micro-riblet array, and micro-pyramid array. ${ }^{187}$ Also, the precision-shaped V-shaped sharp grinding wheel was utilized to process the micro-structure core with controllable shape accuracy and surface quality as the negative template of the finished product. Then, the micro-structured LED diffusion plate was quickly prepared by the micro-imprinting method (Fig. 16(a)). After this method, the test found that the lighting brightness and processing speed are significantly improved..$^{185}$

In addition to cutting the microstructures, high-speed wire electric discharge machining, laser interference lithography processing, and electrolyte jet processing are used to process high-quality microstructures. The method of manufacturing micro-riblet surface structures on metal plates using high-speed wire electric discharge machining (WEDM-HS) was designed and proposed by Chu et al. The surface modified by this method has the advantages of hydrophobicity, low cost, and rapid prototyping. ${ }^{162}$ Bai et al. successfully processed the sub-millimetre micro-grooves of equal width on the parts made of beryllium using electrochemical machining technology ${ }^{186}$ Experiments show that the electrolyte is low-concentration sodium nitrate to make the surface processed micro-grooves smoother (Fig. 16(c)). A focused laser interference lithography processing method with sufficient energy density and high processing efficiency was proposed by $\mathrm{H}$. Wu et al. ${ }^{176}$ The groove structure was processed directly on the metal surface and the processed metal groove has the structural color and anisotropic wettability. Controllable processing and high precision are the advantages of this processing method (Fig. 16(c)). A kind of electrolyte jet processing has been explored that can be used for complex three-dimensional curved surface processing and matrix pits with a diameter of 300 microns, a depth of 20 microns, and a trench structure with a depth of 30 microns are fabricated on the surface of the metal plate and the metal cylinder. ${ }^{188}$ In addition, chemical corrosion to remove the materials to form smoother microgrooves has the potential to be effectively combined with other processing methods. ${ }^{179}$ The material removal process is carried out on a microscale and the formed microstructure has the advantages of no surface cracks, no micro-deformation, and no residual stress. However, the appearance of the spraying and coating processing surfaces makes the formation of the surface features more convenient. In the next section, we will briefly introduce the latest development of coatings in the direction of drag reduction.

\subsection{Coating technology}

The rapid development of coating technology makes it possible to characterize the surface microstructure, such as the processing of compliant and superhydrophobic surfaces. Due to simple manufacturing and installation, coatings are widely applied to change the surface characteristics of materials. ${ }^{29,166}$ Both the drag-reduction surface and the hydrophobic surface are based on the microstructure, and the combined effect of the two has been extensively researched and manufactured. Dou et al. utilized the coating technology to create a bionic surface to reduce the drag. ${ }^{115}$ The coating mixture was applied to the bionic surface of the substrate to form micron-scale caves. Also, experimental tests found that the bionic surface has a visible drag reduction effect. For coating on the microstructure, the effect of the hydrophobic coating on the drag reduction of the riblets was discussed by Abu Rowin et al. ${ }^{189}$ It was found that the hydrophobic coating can effectively assist the drag reduction of the riblets when the spacing of the trench $\mathrm{S}^{+}>30$, and the use of the coating is convenient for the riblet manufacturing (Fig. 17(a)). For compliance coating, Schrader proposed to use polymer materials to spray on the bottom of the ship in order to 


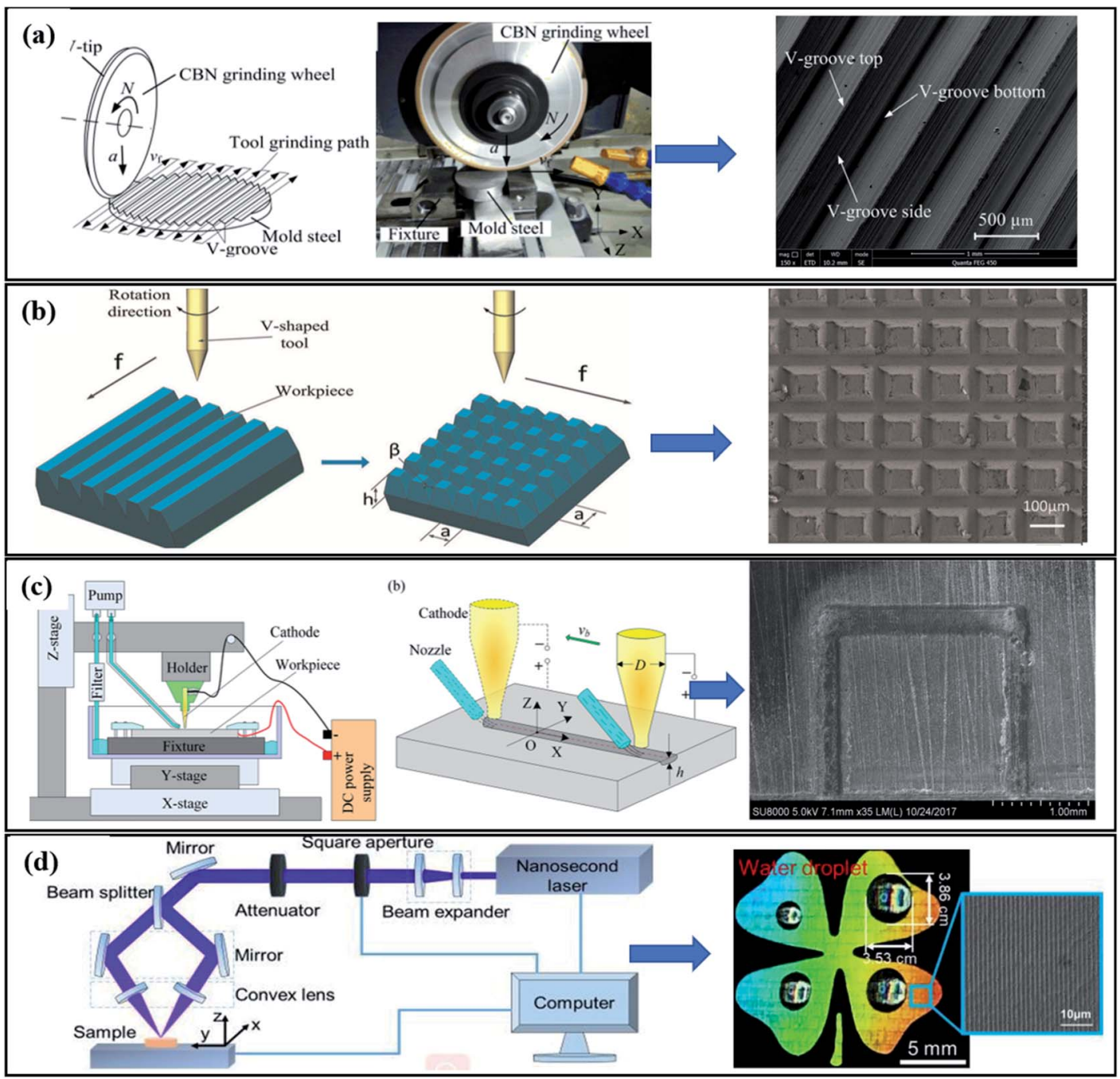

Fig. 16 (a) Precision grinding of the microstructures. Reproduced from ref. 185 with permission from MDPI (Basel, Switzerland), copyright 2020. (b) Schematic diagram of the cutting steps of the micro-textured surface. Reproduced from ref. 145 with permission from SAGE Publications Ltd, copyright 2016. (c) Image of the electrochemical machining method (ECM) to process the sub-millimetre microstructures. Reproduced from ref. 186 with permission from Springer Nature, copyright 2019. (d) Schematic illustration of a laser interference lithography processing method to process the microstructures on the metal surface. Reproduced from ref. 176 with permission from Royal Society of Chemistry, copyright 2019.

form a similar drag reduction coating for dolphins. ${ }^{37}$ The test found that the coating has the drag-reduction effect (Fig. 17(b)).

The riblets with drag reduction ability and the drag reduction agent PAM were tested by the method of UV-induced polymerization (Fig. 17(c)) and it was found that the riblets grafted with the drag-reduction agent had a $14 \%$ reduction in drag compared to the traditional riblet and the drag-reduction performance was maintained for 14 days. ${ }^{170}$ Qin et al. utilized the efficient drag-reduction effect of the shark surface and the hydrophobic capacity of the lotus leaf and proposed a method for manufacturing commercial polyurethane (PU) polymers. ${ }^{64}$ The manufacturing idea is to transfer the microstructure on the sharkskin to the PU through micro-casting technology and then spray the mesoporous silicon microspheres (MSNs) on the PU plate to form a hydrophobic structure. The double-layer structure has been tested to show that the hydrophobic structure is stable and the drag-reduction effect reaches 37\%. Bionic coatings with drag-reduction characteristics inspired by the lubricating properties of fish mucus and waterproof adhesion capabilities were studied by Zhao et al. ${ }^{\mathbf{1 2 7}}$ The drag reduction performance of gel-derived bionic coatings reached $40 \%$ and the adhesion performance to metal substrates increased to 5.35 MPa. The continuous development of the coating technology makes it easier to process the drag-reduction surface. The combination of the coating technology and other processing methods can obtain a more significant drag-reduction effect. Therefore, the combination of multiple processing technologies has become a future trend, and the coating has strong compatibility and has become the preferred method for processing special surfaces.

\subsection{Other methods}

With technological innovation, new methods are gradually being proposed. A method for manufacturing flexible skin with 

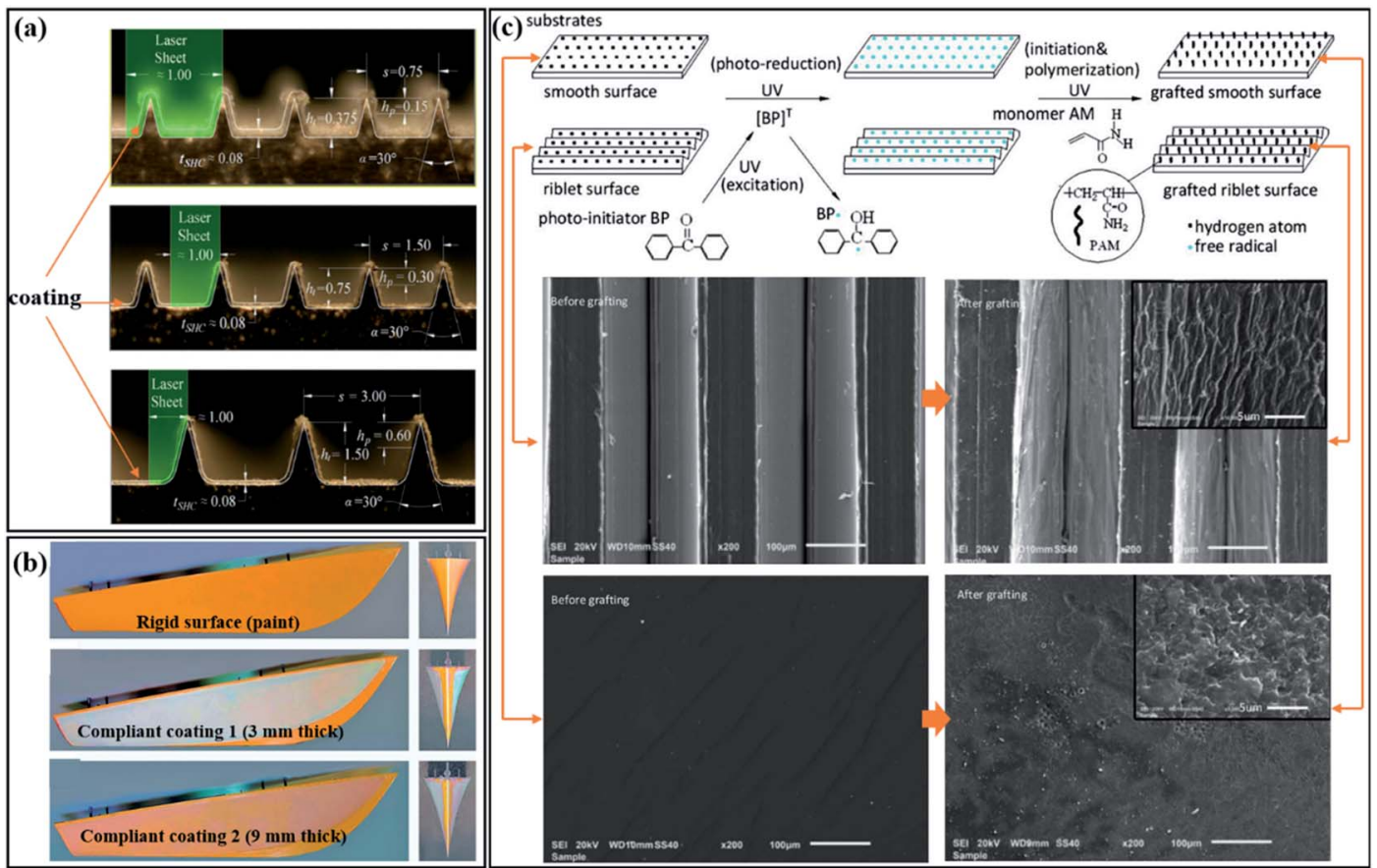

Fig. 17 (a) Image of riblet surfaces with the superhydrophobic coating. Reproduced from ref. 189 with permission from Elsevier, copyright 2018. (b) Schematic diagram of the compliant surface of the bottom of the ship. Reproduced from ref. 37 with permission from Society of Naval Architects and Marine Engineers, copyright 2019. (c) Schematic diagram of smooth and riblet surfaces with UV grafting polymerization. Reproduced from ref. 170 with permission from John Wiley and Sons Inc., copyright 2015.
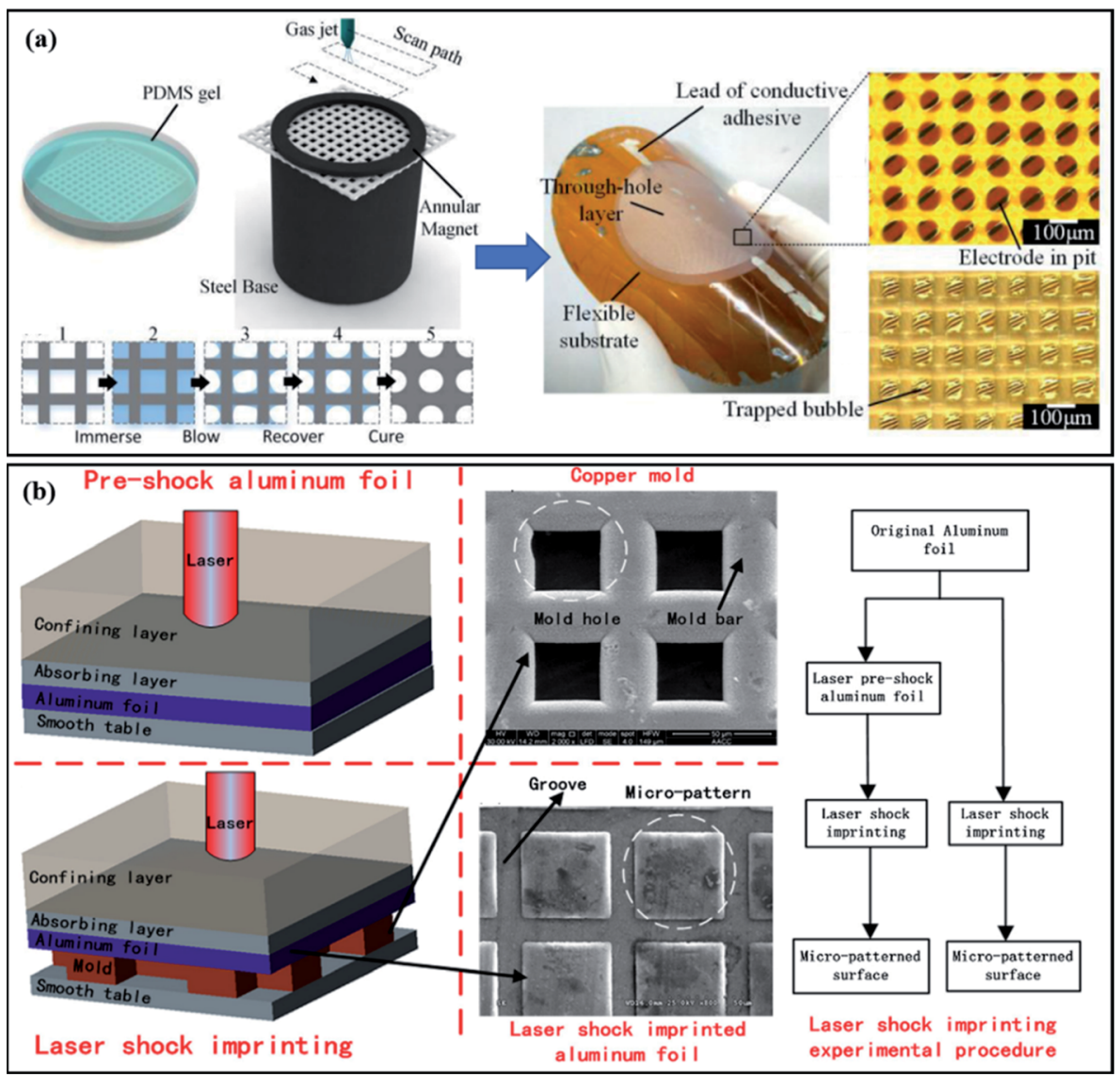

Fig. 18 (a) Image of manufacturing a micro through-hole layer by gas-blowing and the SEM image of a flexible substrate. Reproduced from ref. 190 with permission from IOP Publishing, copyright 2017. (b) Schematic diagram of laser shock micro-pattern imprinting. Reproduced from ref. 181 with permission from Elsevier Ltd, copyright 2019. 

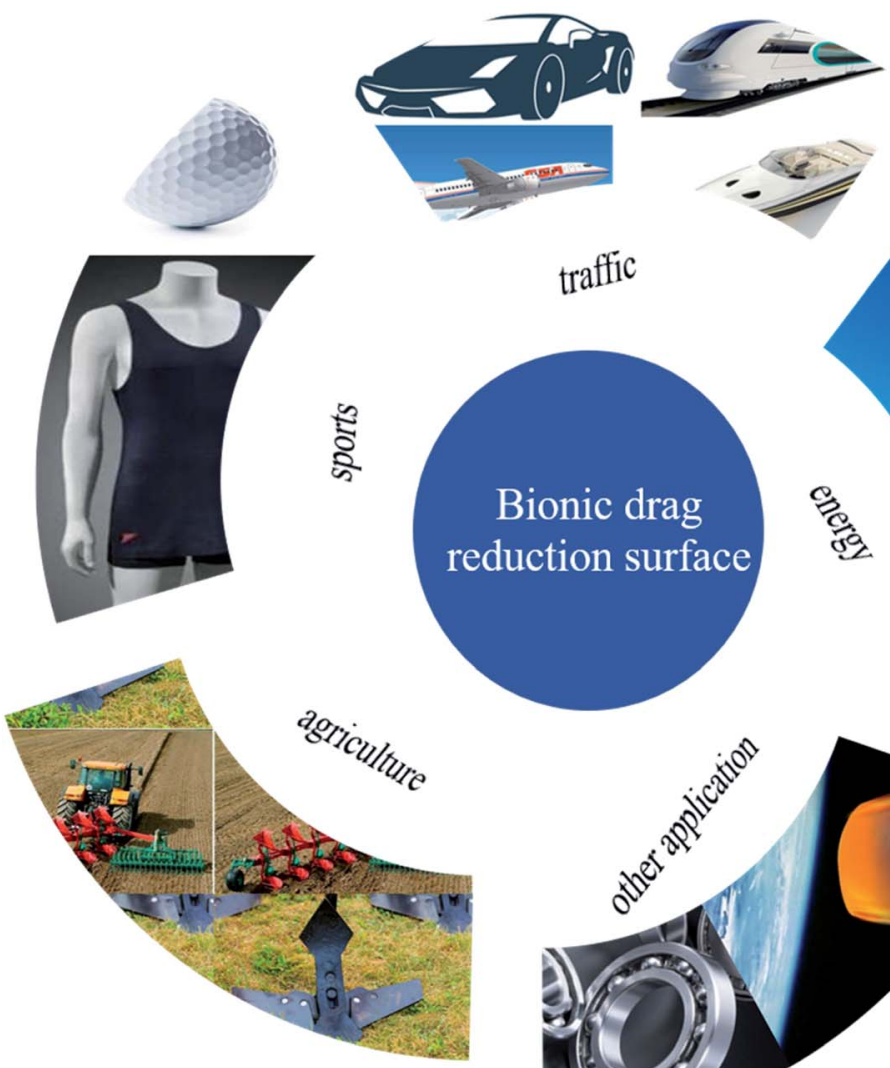

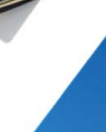
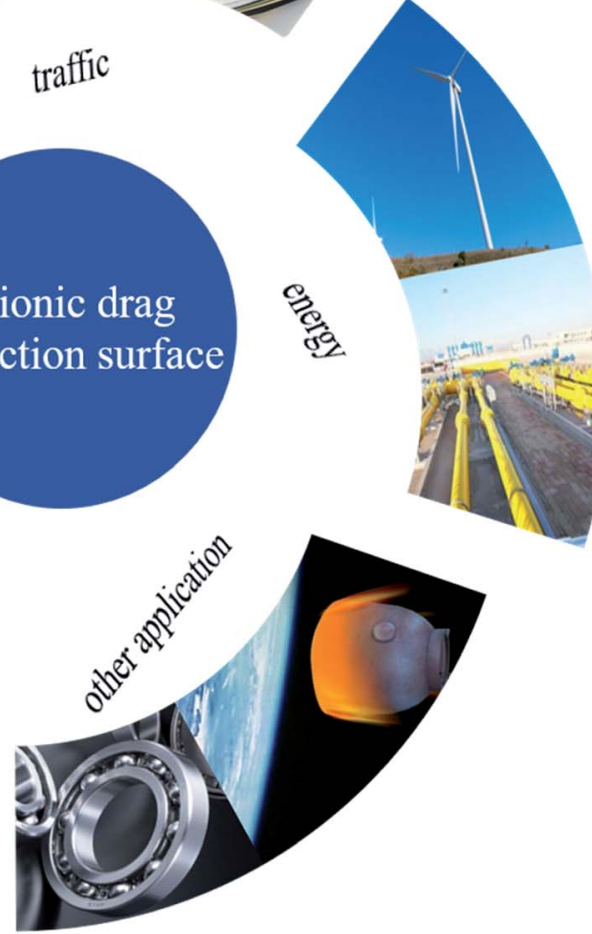

Fig. 19 Application of bionic drag-reduction surfaces (traffic, energy resources, sports, agriculture, and other applications).

the through-hole array by gas-blowing was proposed by $\mathrm{Y}$. Li et al. ${ }^{190}$ Also, the flexible skin with bell-shaped through-hole was found to have $45 \%$ drag-reduction effect through multiphase computational fluid dynamics model simulation (Fig. 18(a)). A processing method utilizing micro-electrical discharge machining (micro-EDM) technology on soft food raw material gelatin gel was proposed by Tamura et al. ${ }^{159}$ This method precisely controls the processing to produce $2 \mu \mathrm{m}$ microgrooves and imprint it on gelatin. The three-dimensional ultrasonic elliptical vibration deformation technology (3D-UEVT) was used to prepare a new diamond-shaped microstructured surface, which improved the wettability performance compared with the plane processed by the cutting method. ${ }^{147}$ Man et al. proposed the use of laser shock imprinting technology to fabricate the micro/nanostructures and the use of laser pre-excitation aluminium foil preparation to improve the residual rough structure and fine defects on the laser shock stamp to prepare a smooth and mechanically good microstructure surface (Fig. 18(b)). ${ }^{181}$ New manufacturing technologies continue to appear with the use of different materials that carry manufacturing technologies and the manufacture of different microstructures so as to suit special environments. The application is different to adapt to the corresponding material; thus, in the next section, the application of the bionic drag-reduction surface will be introduced.

\section{Applications}

Friction that hinders the movement of an object needs to be overcome in most applications. Therefore, drag-reduction surfaces are the most effective way to reduce unfavorable friction and save energy in applications. Moreover, there is a tendency for the microstructure on the drag-reduction surface to affect the overall surrounding environment. In this section, as shown in Fig. 19, we will briefly present the applications in bionic drag-reduction materials, including traffic, energy, sport, and agriculture.

\subsection{Traffic}

The surfaces of vehicles such as boats, trains and aeroplanes are produced in contact with fluids. The surface treatment and application can refer to underwater creatures so as to achieve drag reduction and energy-saving effects. In recent years, machines based on the bionic design of aquatic organisms provide new opportunities for the research and development of the ocean. Aquatic organisms have high manoeuvrability and excellent drag-reduction performance and can be utilized as a template for the development of enhanced bionic vehicle technology. ${ }^{191}$ Weihs utilized a strategy of suddenly accelerating to a higher speed and then gliding during the voyage to save their energy, ${ }^{101}$ and intermittent bionic flight strategy was also discovered by Rayner on migratory bird creatures. ${ }^{9}$ Inspired by this, the climb/glide strategy was utilized to evaluate the 
distance travelled by aircraft. The results show that the energysaving effect is more than $10 \%-40 \%$ relative to the horizontal flight transition. ${ }^{7}$ The current underwater vehicle technology has limitations of speed, efficiency, manoeuvrability, and stealth. The biological performance of the biological autonomous underwater vehicles (BAUV) technology mentioned by Frank E. Fish ${ }^{99}$ generally exceeds the existing engineering performance.

New vehicle solutions developed using bionic design can significantly improve their speed, efficiency, and mobility. The bionic dolphin robot designed by $\mathrm{Yu}$ et al. more realistically restores the underwater propulsion of dolphins and novelty combines the propulsion methods of real dolphins and traditional underwater gliders. ${ }^{192}$ The developed dolphin-style gliding robot has powerful movement capabilities and manoeuvrability, which provides the prospect of expanding the scope of underwater detection and work. ${ }^{193}$ Inspired by the biology of a body's caudal fin capillary-like fish propulsion mechanism, Chowdhury et al. created a two-joint, three-link multi-body vehicle model and numerically analyzed it during straight-line navigation. ${ }^{194} \mathrm{~A}$ V-shaped riblet completely covered the airfoil and was tested in a wind tunnel. ${ }^{177}$ It was found that a drag reduction effect of about $6 \%$ was produced. Besides, the $\mathrm{V}$-shaped riblet structure replicating the shark skin can be used on the surface of the aircraft wing to achieve drag reduction and to increase the sailing distance. Also, the bionic structure is used to optimize the key mechanical components, and the biooptimized drag reduction effect of the twin-turbo torque converter (YJSW335) is realized. Fish-shaped blades, dolphinshaped turbine, and stator, the bionic structure of the nonsmooth surface of the pump make the machine have excellent efficiency. ${ }^{195}$ Regardless of whether the streamlined shape or the surface microstructure is used in vehicles to make the transportation more efficient, the energy can also be rationally used on the bionic surface to reduce drag.

\subsection{Energy sources}

With the emergence of the energy crisis, it is urgent to evolve a batch of technologies that can efficiently solve energy waste; the use of bionic surface technology will bring new opportunities and development. Pipeline transportation has the characteristics of continuous transportation, large transportation volume, and high efficiency, which is the most common transportation method in natural gas and oil. The bionic surface is utilized to further increase the transportation efficiency and save energy loss. W. Liu et al. proposed a new type of pipe surface shape with lateral micro-grooves to reduce the frictional loss of fluid transported by pipelines. ${ }^{150}$ The internal vortex generated on the micro-groove surface of the bionic pipeline is a key factor in drag reduction. Also, the maximum drag reduction rate in the water tunnel experiment is $3.21 \%$. A bionic pipeline can provide a reference for energy saving in pipeline transportation. Further, to promote the closed channel, the low drag riblet structure inspired by shark skin is utilized in the closed channel. The experiment tested by Büttner and Schulz found that the riblet structure at the bottom is better than the other surfaces in the turbulent flow. ${ }^{\mathbf{1 9 6}}$ The optimum dimensionless riblet spacing value at $S^{+} \approx 15$ and $100 \mu \mathrm{m}$ sawtooth samples can provide a maximum pressure drop reduction of $26 \%$.

During pipeline transportation, coatings, biomimetic microstructures, and injecting nano-chain drag reduction additives have been applied and have achieved good results. ${ }^{197}$ Both the compliant coating stimulated by dolphins and the shark scales-like microstructure surface has been tested by a variety of methods to verify its practicality. And, the use of the drag-reducing additive has more potential due to the superefficient drag reduction rate in pipeline transportation. However, the combination of a variety of drag-reduction methods is the future development direction because it can achieve a higher drag reduction rate. The integration of biocomposite shark skin and polymer additives was proposed by Chen et al., which was experimentally found to have a high dragreduction effect of $80 \% .{ }^{\mathbf{1 6 9}}$ The drag-reducing additive is added to change the characteristics of the fluid inside the pipeline and mucus is considered as a natural drag-reducing additive, which is recognized to absorb the turbulent energy near the surface of the fluid, thereby reducing the force exerted on the surface. The absorbed energy is then dissipated into the bulk fluid, minimizing local fluid turbulence on the wall to reduce friction. The addition of the polymer inhibits the fluid's radial pulsation and reduces the energy consumption. ${ }^{169,198,199}$ The surface of the hierarchical comb-shaped hydrophilic polymer brush (HCHPB) design based on fish mucus was proposed by $\mathrm{Su}$ et al. ${ }^{152}$ The surface has nanostructures that have not only good lubricating properties and drag reduction properties but also have good anti-biological adhesion properties, which provide new ideas for self-cleaning and preventing microbial adhesion.

In addition, for motor blades, inspired by bionics, ${ }^{160} \mathrm{Zhu}$ and Gao utilized a winged propeller to suppress the generation of tip vortex cavitation (TVC). ${ }^{45}$ Also, the simulation results showed that the winglet propeller significantly weakened the strength of the blade tip vortex wake and had a significant effect. Moreover, inspired by the shark skin effect, Büttner and Schulz prepared a riblet structure surface with a fluid wall friction reduction of $4.9 \%{ }^{155}$ A new application of the riblet surface was carried out on the blade of the jet engine and the preparation method of high-temperature resistant nickel alloy was explored, which has an anti-oxidation effect and reduces drag.

\subsection{Sports}

Sports' competitions are very popular because of their viewing ability. Nowadays, outstanding athletes keep setting new records. Therefore, the development of bionic surface technology also extends to the sports field and helps athletes breakthrough one limit after another. In sports competitions, increasing the flying distance of golf and the prohibition of bionic swimsuits illustrate the advantages of bionic surfaces. ${ }^{\mathbf{1 6 5}}$ Alam et al. explored the aerodynamic characteristics and flow pattern of the arc groove on the golf ball. ${ }^{82,83}$ The arc groove structure has a positive effect on the golf ball's flight distance and lifts coefficient. Also, bionics reveals more biological 
surface details and also inspires smart fabric design. ${ }^{\mathbf{2 0 0}}$ For swimsuits, a high-tech swimsuit that replicates shark skin was banned from competitions in 2010 due to its significantly improved performance (Spitz won 7 gold medals in tight underwear at the Athens Olympics and Phelps won 14 gold medals in a high-tech full-body swimsuit at the Beijing Olympics). ${ }^{201}$ Thus, the drag reduction effect of the bionic shark skin drag-reduction swimsuit can be seen. Bionic surfaces still have advantages in antibacterial and anti-fouling, and bionics and multidisciplinary cross-discipline may become the future trend.

\subsection{Agriculture}

Agriculture is the foundation of development and provides basic industries that support the construction and development of the national economy. The optimization of agricultural machinery can also utilize bionic microstructures. Intelligent agriculture created by the combination of bionics and agriculture has advantages in increasing the farming efficiency and reducing labor burden. Ever since human beings entered the farming society, they have been fighting against the adverse effects of soil compaction on the soil. The increase in crop growth and yield has changed by the change in the subsoiler. Therefore, the bionic structure optimization subsoiler highlights its unique advantages, reduces mechanical resistance, fuel consumption, and increases soil disturbance to affect the yield of the cultivated land. For improving the heavy tillage drag during agricultural practice, a biomimetic shark-inspired (BiOS) subsoiler utilizing the riblet structure extracted from shark scales was proposed by Y. Wang et al. ${ }^{\mathbf{2 0 2}}$ The experiment of biomimetic shark-inspired (BiOS) subsoiler found that the drag is the least when the riblet height $h=5 \mathrm{~mm}$ and the ratio of the riblet height to the riblet spacing $h / s=0.57$, which increases the tillage efficiency and improves soil quality. The bionic structure shows attractive application prospects in soil improvement and efficiency in practical applications, which provides new directions for technological breakthroughs.

\subsection{Other application}

The bionic structure also has a good guiding significance in new fields, such as noise reduction, wear resistance, and heat dissipation. Sharks and dolphins have evolved scales and flexible skin during the fight against turbulence, which is considered to be the main target of reverse engineering mechanism drag and noise reduction. ${ }^{92}$ Also, drag reduction, on the other hand, brings another application, which is noise reduction. Noise reduction can reduce environmental pollution and unnecessary vibration. The structure of spanwise riblet inspired by sharkskin has been applied to simulate the hydrodynamic noise of the three-dimensional (3D) hydrofoil and the maximum noise reduction was observed at $7.28 \mathrm{~dB} .{ }^{84}$ The blade bionic groove design proposed by J. Wang, Nakata, and Liu ${ }^{203}$ was applied to the mixed flow fan and it was found that the use of the groove structure has the potential to suppress noise. When the aerodynamic efficiency loss is only 0.3 percentage points, the blade leading edge reduces the turbulent flow energy by about $38 \%$. After optimization, the bionic blade can bring about a large reduction in the noise with a small increase in the drag, thus effectively reducing the noise without affecting the aerodynamic efficiency.

The surface microstructure has the potential to improve the tribological properties and catalyst loading. For wear drag, the surface microstructures can capture the wear particles to reduce further wear in the boundary lubrication. ${ }^{204}$ Ibatan et al. reviewed the potential impact of surface deformation on the tribological properties. ${ }^{205}$ Bionic surface textures were used on bearing sliders to reduce the friction and improve the loadcarrying capacity. For friction reduction, micro-structured microcavity as a fluid-lubricated reservoir can maintain the state of reduced drag more lastingly in hydrodynamic and mixed lubrication. The porous structure mentioned by Chaowei Huang and $\mathrm{GuO}^{206}$ is filled with lubricating oil and stored to improve the friction performance of the bionic surface and increase the wear life. For catalyst loading, the large surface area of the microgrooves facilitates the adhesion of the catalyst and is widely applied.

Besides, the riblet coating inspired by shark skin can also be applied in the aerospace industry to optimize the high temperature in the engine and has an anti-oxidant effect, which can additionally reduce surface skin drag. ${ }^{155}$ The most effective cooling method to protect parts from ablation in an extremely high-temperature environment is transpiration cooling. In practical applications, the use of bionic surfaces increases the surface protective film and obtains a significantly better cooling performance. ${ }^{\mathbf{2 0 7}}$ Gan Huang, Zhu and Liao utilized the bionic non-smooth surface to increase the thickness of the transpiration cooling protective film by $22.7 \%^{208}$ and the transpiration cooling efficiency is significantly increased by $12 \%$. The introduction of bionic structures has broken through the biggest limitations in many fields. Therefore, bioinspired materials may help to overcome the shortcomings in a single field and show unique advantages in practical applications.

\section{Conclusions and perspectives}

Drag reduction is a very desirable feature in many applications. In this review, taking aquatic animals as the main research object, the development of related aquatic animals-inspired drag-reduction technology research is introduced, from which some questions and conjectures are discussed next.

Bionic drag reduction extends the limitation of traditional drag reduction in many aspects and its effect is obvious. For the mechanism explanation of theoretical research, drag reduction under laminar flow has many mechanism explanations supports, such as the protruding height theory. However, in turbulent flow, fluid motion is disordered and complex, and most researchers focus on the effect of drag reduction, while the mechanism of drag reduction is rarely discussed. So far, drag reduction under turbulence is still a hot and difficult topic. The "water-trapping" effect and the 'secondary vortex' effect from the perspective of the vortex are utilized to explain surface drag reduction. Furthermore, boundary layer theory and separation control under reverse pressure conditions have been extensively studied to explore the reasons for drag reduction. 
The results are now mainstream view aquatic animals in water and low resistance non-smooth surface and mucus stealth effect with combined effects. The preparation of bionic drag reduction materials utilizes this point and the composite drag reduction technology also implies the same feature. The preparation of drag-reduction materials combined with matching technology or methods seems to be the trend of future development. For example, lubricant injection method and dragreducing porous material that can store lubrication for a long time, ${ }^{206}$ the storage of the porous surface, and the lubrication of the lubricant are superimposed without affecting each other, making the lubricant more durable, and hydrophobic materials combined with artificial micro/nano-engine technology to improve the efficiency and speed of the micro device. ${ }^{\mathbf{2 0 9 , 2 1 0}}$ Moreover, the application of bionic drag-reduction materials is inseparable from the development of drag reduction technology and can better compensate for each other's defects. Therefore, the two complement each other, gradually becoming the future trend in the field of underwater bionic drag reduction.

The drag-reduction effect of the bionic shark scales is only significant under certain conditions. If the flow field environment, arrangement spacing, and shield scale size are changed, the drag-reduction effect will be weakened or even not. Regardless of whether the shark scale is actively controlled or passively controlled, it can be concluded that the shark scale can swing at an angle of $50^{\circ}$ and the dolphin's corrugated skin during high-speed movement. ${ }^{27}$ Compared with the fixed shark scales in the experiment, it seems that the shark scales can be regarded as continuously adjusting to the flow field and the imitation sample fixed in the experiment seems to be a moment during the movement of the shark scales. Thus, the common features of drag-reduction bionic surface need to be further explored. The extent to which the scales are regarded as dynamic on skin hydrodynamic function is unclear but it is a promising field to link skin structure with locomotor hydrodynamics. ${ }^{2}$

Aquatic animals, as a research object of bionic dragreduction, have different morphological characteristics, such as the volume, surface texture, and posture of aquatic animals, and their adaptability to the flow field. The color pattern seems to have a potential mechanism for underwater creatures to resist water flow. ${ }^{\mathbf{1 0 8}}$ During the fight between underwater creatures and water flow, a more organized skin pattern has evolved, which vibrates slightly during swimming. ${ }^{92}$ For the experimental setup, more abundant measurement techniques and visualization methods for later treatment of the flow field structure can be adopted. The visual treatment of vortices is conducive for the analysis of the generation, development, and extinction of vortices in the boundary layer. Color is introduced into PIV measurement of the boundary layer microbubbles and tomographic particle image velocimetry (TPIV) measurement. ${ }^{81,211}$ Also, the measurement of aquatic animals hydrodynamic parameters is jointly verified by experiment and computer simulation. The surface function of aquatic animals in the body during movement is rarely replicated with experimental models at present.
Bionic manufacturing is more inclined to replicate the surface of bionic microstructures, which is limited by processing materials and manufacturing processes. Large-scale manufacturing of drag-reduction surfaces is still the key point to be explored in the future. Combining new materials and advanced manufacturing processes will be a breakthrough in drag reduction preparation. For example, the combination of coatings and grooves forms a self-cleaning surface, the surface of the wear-resistant structure is combined with the hydrophobic to form a wear-resistant hydrophobic surface, and micro-device process manufacturing with drag-reduction materials to drive the application of targeted drugs, etc. In addition, bionic drag-reduction materials have broad prospects in anti-fouling, anti-fog, water collection, wear resistance, micro-engine drag reduction, heat dissipation, catalysis, etc.

To sum up, although aquatic organisms have developed a variety of materials, surfaces, and structures ranging from macroscopic to nanoscale, using which as imitation objects to solve engineering problems has brought us advanced templates, the close integration of multiple disciplines is still one of the future development trends. Thus, underwater bionic drag-reduction materials still urgently need further exploration and research in the future.

\section{Conflicts of interest}

There are no conflicts to declare.

\section{Acknowledgements}

This work is supported by the National Natural Science Foundation of China (No. 52005227), the Opening Project of the Key Laboratory of Bionic Engineering (Ministry of Education), Jilin University (No. KF20200007) and the Natural Science Research Project of Jiangsu Province University (No. 20KJB410003).

\section{References}

1 J. C. Liao, J. Exp. Biol., 2006, 209, 4077-4090.

2 G. V. Lauder, D. K. Wainwright, A. G. Domel, J. C. Weaver, L. Wen and K. Bertoldi, Phys. Rev. Fluids, 2016, 1, 1-18.

3 A. W. Lang, E. M. Jones and F. Afroz, Bioinspiration Biomimetics, 2017, 12, 026005.

4 F. B. Tian, Comput. Mech., 2019, 65, 751-761.

5 F. Afroz, A. Lang, M. L. Habegger, P. Motta and R. Hueter, Bioinspiration Biomimetics, 2017, 12, 016009.

6 Z. Liu, W. Yin, D. Tao and Y. Tian, Biotribology, 2015, 1-2, 11-23.

7 T. F. Miller, Ocean Eng., 2018, 149, 78-94.

8 J. M. van der Hoop, M. L. Byron, K. Ozolina, D. L. Miller, J. L. Johansen, P. Domenici and J. F. Steffensen, J. Exp. Biol., 2018, 221, 1-11.

9 J. M. V. Rayner, J. Theor. Biol., 1985, 117, 47-77.

10 W. Li, D. Roggenkamp, T. Hecken, W. Jessen, M. Klaas and W. Schröder, Exp. Fluids, 2018, 59, 1-18.

11 T. Fletcher, J. Altringham, J. Peakall, P. Wignall and R. Dorrell, Proc. R. Soc. B, 2014, 281, 20140703. 
12 J. C. Liao, D. N. Beal, G. V. Lauder and M. S. Triantafyllou, J. Exp. Biol., 2003, 206, 1059-1073.

13 J. C. Liao, J. Exp. Biol., 2004, 207, 3495-3506.

14 K. B. Golovin, J. Gose, M. Perlin, S. L. Ceccio and A. Tuteja, Philos. Trans. R. Soc., A, 2016, 374, 20160189.

15 G. Liu, Z. Yuan, Z. Qiu, S. Feng, Y. Xie, D. Leng and X. Tian, Ocean Eng., 2020, 199, 106962.

16 Y. Sun and Z. Guo, Nanoscale Horiz., 2019, 4, 52-76.

17 M. Perlin, D. R. Dowling and S. L. Ceccio, J. Fluids Eng., 2016, 138, 091104.

18 T. L. Daniel, Biol. Bull., 1981, 160, 376-382.

19 K. Yanase and P. Saarenrinne, J. Exp. Biol., 2015, 218, 13731385.

20 A. W. Lang, M. T. Bradshaw, J. A. Smith, J. N. Wheelus, P. J. Motta, M. L. Habegger and R. E. Hueter, Bioinspiration Biomimetics, 2014, 9, 036017.

21 A. W. Lang, E. M. Jones and F. Afroz, Bioinspiration Biomimetics, 2017, 12, 026005.

22 D. W. Bechert, M. Bruse, W. Hage and R. Meyer, Naturwissenschaften, 2000, 87, 157-171.

23 F. Afroz, M. A. R. Sharif and A. Lang, J. Fluids Struct., 2016, 62, 187-208.

24 Y. Cai, L. Lin, Z. Xue, M. Liu, S. Wang and L. Jiang, Adv. Funct. Mater., 2014, 24, 809-816.

25 Z. Wang, S. Ma, H. Zhong, W. Yang, X. Pei, B. Yu, K. Su and F. Zhou, Mater. Today Commun., 2019, 21, 100618.

26 I. G. Nesteruk, Int. J. Fluid Mech. Res., 2014, 41, 260-281.

27 F. Afroz, A. Lang, M. L. Habegger, P. Motta and R. Hueter, Bioinspiration Biomimetics, 2017, 12, 016009.

28 F. Afroz, A. Lang, M. L. Habegger, P. Motta and R. Hueter, Bioinspiration Biomimetics, 2016, $12(1), 016009$.

29 H. B. Evans, A. M. Hamed, S. Gorumlu, A. Doosttalab, B. Aksak, L. P. Chamorro and L. Castillo, Proc. Natl. Acad. Sci. U. S. A., 2018, 115, 1210-1214.

30 W. Sagong, C. Kim, S. Choi, W. P. Jeon and H. Choi, Phys. Fluids, 2008, 20, 101510.

31 S. Van Wassenbergh, K. Van Manen, T. A. Marcroft, M. E. Alfaro and E. J. Stamhuis, J. R. Soc., Interface, 2015, 12, 20141146.

32 H. Ferrón, C. Pla, C. Martínez-Pérez, M. J. Escudero-Mozo and H. Botella, J. Iber. Geol., 2014, 40, 87-97.

33 E. M. Dillon, R. D. Norris and A. O'Dea, Mar. Ecol.: Prog. Ser., 2017, 566, 117-134.

34 G. Fiore, E. Anderson, C. S. Garborg, M. Murray, M. Johnson, M. J. Moore, L. Howle and K. A. Shorter, PLoS One, 2017, 12, 1-19.

35 R. W. Blob, W. C. Bridges, M. B. Ptacek, T. Maie, R. A. Cediel, M. M. Bertolas, M. L. Julius and H. L. Schoenfuss, Integr. Comp. Biol., 2008, 48, 734-749.

36 A. W. Lang, P. Motta, P. Hidalgo and M. Westcott, Bioinspiration Biomimetics, 2008, 3, 046005.

37 L. U. Schrader, J. Shellfish Res., 2019, 63, 206-218.

38 K. S. Yeo, AIAA J., 1998, 36, 656-658.

39 M. Muthuramalingam, L. S. Villemin and C. Bruecker, J. Exp. Biol., 2019, 222, jeb205963.

40 L. Wu, Z. Jiao, Y. Song, C. Liu, H. Wang and Y. Yan, Sci. Rep., 2018, 8, 1-8.
41 L. Y. Wu, Z. Bin Jiao, Y. Q. Song, W. T. Ren, S. C. Niu and Z. W. Han, Sci. China: Technol. Sci., 2017, 60, 1111-1117.

42 G. Q. Yan, P. Tian, J. S. Mo, H. Xie and H. L. Wei, Int. J. Agric. Biol. Eng., 2020, 13, 7-14.

43 L. Wen, J. C. Weaver and G. V. Lauder, J. Exp. Biol., 2014, 217, 1656-1666.

44 F. E. Fish and G. V. Lauder, Annu. Rev. Fluid. Mech., 2006, 38, 193-224.

45 W. Zhu and H. Gao, J. Mar. Sci. Eng., 2019, 7, 0333.

46 A. G. Domel, M. Saadat, J. C. Weaver, H. Haj-Hariri, K. Bertoldi and G. V. Lauder, J. R. Soc., Interface, 2018, 15, 1-9.

47 J. Wang, C. Pan, Q. Zhang and T. Li, AIAAJ., 2018, 56, 26422652.

48 P. R. Bandyopadhyay, Integr. Comp. Biol., 2002, 42, 102-117. 49 J. D. Ott, M. T. Lazalde and G. Gu, Bioinspiration Biomimetics, 2019, 15, 026001.

50 K. T. Du Clos, A. Lang, S. Devey, P. J. Motta, M. L. Habegger and B. J. Gemmell, J. R. Soc., Interface, 2018, 15, 14-18.

51 G. Díez, M. Soto and J. M. Blanco, J. Fish Biol., 2015, 87, 123137.

52 A. Lang, P. Motta, M. L. Habegger, R. Hueter and F. Afroz, Mar. Technol. Soc. J., 2011, 45, 208-215.

53 J. Oeffner and G. V. Lauder, J. Exp. Biol., 2012, 215, 785-795.

54 F. E. Fish, P. Legac, T. M. Williams and T. Wei, J. Exp. Biol., 2014, 217, 252-260.

55 S. R. Noren, J. V. Redfern and E. F. Edwards, J. Exp. Biol., 2011, 214, 4151-4159.

56 V. V. Pavlov, Bioinspiration Biomimetics, 2006, 1, 31-40.

57 F. E. Fish, Bioinspiration Biomimetics, 2006, 1, R17-R25.

58 U. Ehrenstein and C. Eloy, J. Fluid Mech., 2013, 718, 321346.

59 M. Trassinelli, J. Theor. Biol., 2016, 396, 42-52.

60 P. W. Carpenter, Flow Phenom. Nat. Vol. 2, 2006, vol. 4, pp. 447-456.

61 V. M. Kulik, A. V. Boiko and I. Lee, Thermophys. Aeromechanics, 2018, 25, 515-524.

62 D. K. Wainwright, F. E. Fish, S. Ingersoll, T. M. Williams, J. St Leger, A. J. Smits and G. V. Lauder, Biol. Lett., 2019, 15, 9-14.

63 D. August, D. Gino, J. C. Weaver, S. Mehdi, B. Katia and G. V. Lauder, Bioinspiration Biomimetics, 2018, 151, 1-34.

64 L. Qin, M. Hafezi, H. Yang, G. Dong and Y. Zhang, Micromachines, 2019, 10, 490.

65 M. V. Ankhelyi, D. K. Wainwright and G. V. Lauder, J. Morphol., 2018, 279, 1132-1154.

66 M. R. Raupach, R. A. Antonia and S. Rajagopalan, Appl. Mech. Rev., 1991, 44(1), 1.

67 J. Jim, Annu. Rev. Fluid. Mech., 2004, 36, 173-196.

68 M. J. Walsh and A. M. Lindemann, AIAA , 1984, 0347.

69 S. Martin and B. Bhushan, J. Colloid Interface Sci., 2016, 474, 206-215.

70 P. Luchini, Eur. J. Mech. B/Fluids, 2018, 71, 15-34.

71 K. A. Flack and M. P. Schultz, Phys. Fluids, 2014, 26, 101305.

72 M. P. Schultz and K. A. Flack, Phys. Fluids, 2009, 21, 015104.

73 G. Cui, C. Pan, D. Wu, Q. Ye and J. Wang, Chin. J. Aeronaut., 2019, 32, 2433-2442. 
74 S. Martin and B. Bhushan, J. Fluid Mech., 2014, 756, 5-29.

75 B. Dean and B. Bhushan, Philos. Trans. R. Soc., A, 2010, 368, 4775-4806.

76 D. W. Bechert and M. Bartenwerfer, J. Fluid Mech., 1989, 206, 105-129.

77 D. W. Bechert and G. Hoppe, AIAA, 1985, 3, 54-67.

78 A. Bottaro, J. Fluid Mech., 2019, 877, P1.

79 C. Huang, D. Liu and J. Wei, Chem. Eng. Sci., 2016, 152, 267279.

80 S. K. Robinson, Annu. Rev. Fluid Mech., 1991, 56, 601-639.

81 S. Q. Yang, S. Li, H. P. Tian, Q. Y. Wang and N. Jiang, Acta Mech. Sin., 2015, 32, 284-294.

82 K. Aoki, K. Muto and H. Okanaga, Procedia Eng., 2010, 2, 2431-2436.

83 F. Alam, T. Steiner, H. Chowdhury, H. Moria, I. Khan, F. Aldawi and A. Subic, Procedia Eng., 2011, 13, 226-231.

84 Z. Dang, Z. Mao and W. Tian, J. Mar. Sci. Eng., 2019, 7, 136.

$85 \mathrm{~F} . \mathrm{Xu}, \mathrm{S}$. Zhong and S. Zhang, Phys. Fluids., 2018, 30, 051901.

86 X. Pu, G. Li and H. Huang, Biol. Open, 2016, 5, 389-396.

87 X. Han, D. Y. Zhang, X. Li and Y. Y. Li, Chin. Sci. Bull., 2008, 53, 1587-1592.

88 S. B. Creager and M. E. Porter, Zoology, 2018, 126, 154-163. 89 G. X. Joshua Ott and M. Lazalde, Mater. Res. Express, 2019, 0-12.

90 L. Wen, J. C. Weaver, P. J. M. Thornycroft and G. V. Lauder, Bioinspiration Biomimetics, 2015, 10, 66010.

91 L. Li, C. Beall, K. Piipari, H. Al-qassab and M. A. Smith, Biochem. J., 2019, 0-14.

92 P. R. Bandyopadhyay and A. M. Hellum, Sci. Rep., 2014, 4, 6650.

93 B. Y. J. Gray, J. Exp. Biol., 1936, 13, 192-199.

94 M. Gad-el-Hak and D. M. Bushnell, J. Fluids Eng., 1991, 113, 5-30.

95 P. L. A. Carpenter, Currentence, 2000, 79, 758-765.

96 Z. Wang, K. S. Yeo and B. C. Khoo, Eur. J. Mech. B/Fluids, 2006, 25, 33-58.

97 T. Von Kramer, Nav. Eng. J., 1956, 341-348.

98 T. K. Von, ASNE J., 1961, 103-108.

99 F. E. Fish, Chem. Eng. J., 2019, 5, 0-35.

100 D. B. Nagel, W. E. Resch and M. M. Kröner, High Perform. Comput. Sci. Eng., 2013, pp. 379-391, 9783642238.

101 D. Weihs, Integr. Comp. Biol., 2002, 42, 1071-1078.

102 S. H. Ridgway and D. A. Carde, IEEE Eng. Med. Biol. Mag., 1993, 12, 83-88.

103 T. I. Józsa, E. Balaras, M. Kashtalyan, A. G. L. Borthwick and I. M. Viola, J. Fluid Mech., 2019, 866, 689-720.

104 V. L. Lorang, B. Podvin and P. Le Quéré, Phys. Fluids, 2008, 20, 75-110.

105 S. Kunze and C. Brücker, Exp. Fluids, 2011, 50, 747-759.

106 B. J. McKeon, I. Jacobi and S. Duvvuri, AIAA J., 2018, 56, 2178-2193.

107 M. Hassanalian, H. Abdelmoula, S. Ben Ayed and A. Abdelkefi, J. Therm. Biol., 2017, 66, 27-32.

108 M. Hassanalian, H. Abdelmoula, S. Mohammadi, S. Bakhtiyarov, J. Goerlich and U. Javed, J. Therm. Biol., 2019, 84, 292-310.
109 L. Tian, Y. Wang, Z. Li, H. Mei and Y. Shang, Exp. Therm. Fluid Sci., 2017, 85, 363-369.

110 F. C. Li, Y. Kawaguchi and K. Hishida, Phys. Fluids, 2004, 16, 3281-3295.

111 J. W. R. Peeters, R. Pecnik, M. Rohde, T. H. J. J. Van Der Hagen and B. J. Boersma, J. Fluid Mech., 2016, 799, 505-540.

112 J. C. Liao, Philos. Trans. R. Soc., B, 2007, 362, 1973-1993.

113 G. Novati, S. Verma, D. R. Dmitry Alexeev, W. M. van Rees and P. Koumoutsakos, Bioinspiration Biomimetics, 2017, 12, 036001.

114 E. M. Quicazan-Rubio, J. L. Van Leeuwen, K. Van Manen, M. Fleuren, B. J. A. Pollux and E. J. Stamhuis, J. R. Soc., Interface, 2019, 16, 20180714.

115 Z. Dou, J. Wang and D. Chen, J. Bionic Eng., 2012, 9, 457464.

116 H. Zhou, C. Liu, G. Tian, X. Feng and C. Jia, Microsc. Res. Tech., 2020, 1-9.

117 H. Zhou, J. Cui, G. Tian, Y. Zhu and C. Jia, Adv. Mech. Eng., 2020, 12, 1-10.

118 M. Uehara, K. Taguchi, T. Imagawa and H. Kitagawa, J. Morphol., 2000, 244, 15-22.

119 M. S. Byeon, J. Y. Park, S. W. Yoon and H. W. Kang, J. Appl. Ichthyol., 2011, 27, 67-72.

120 S. J. Lee, H. N. Kim, W. Choi, G. Y. Yoon and E. Seo, Soft Matter, 2019, 15, 8459-8467.

121 D. K. Wainwright and G. V. Lauder, Zoology, 2016, 119, 182195.

122 M. W. N. Rosen and N. E. Cornford, Nature, 1971, 234, 4951.

123 H. A. Abdulbari, F. W. M. Ling, Z. Hassan and H. J. Thin, Adv. Polym. Technol., 2018, 37, 3136-3145.

124 N. K. Jha, A. Bhatt and R. N. Govardhan, Exp. Fluids, 2019, 60, 1-18.

125 R. A. Verschoof, R. C. A. Van Der Veen, C. Sun and D. Lohse, Phys. Rev. Lett., 2016, 117, 1-4.

126 S. Elghobashi, Annu. Rev. Fluid. Mech., 2019, 51, 217-244.

127 W. Zhao, R. Xu, L. Zhang, Y. Zhang and Y. Wang, Prog. Org. Coat., 2020, 139, 105442.

128 K. Luo, Q. Dai, X. Liu and J. Fan, Int. J. Multiphase Flow, 2019, 111, 140-157.

129 T. C. Corke and F. O. Thomas, AIAA J., 2018, 56, 3835-3847.

130 G. Bernadsky, N. Sar and E. Rosenberg, J. Fish Biol., 1993, 797-800.

131 K. L. Shephard, Rev. Fish Biol. Fish., 1994, 4, 401-429.

132 Y. Luo and D. Zhang, Appl. Mech. Mater., 2011, 44-47, 11511157.

133 T. W. Kim, J. Nanosci. Nanotechnol., 2014, 14, 7562-7568.

134 H. Chen, X. Zhang, D. Zhang, J. Pan and I. Hagiwara, J. Appl. Polym. Sci., 2013, 130, 2383-2389.

135 H. Chen, D. Che, X. Zhang, Y. Yue and D. Zhang, J. Micromech. Microeng., 2015, 25, 017002.

136 Y. Luo, Y. Liu, J. Anderson, X. Li and Y. Li, Appl. Phys. A: Mater. Sci. Process., 2015, 120, 369-377.

137 T. Kim, R. Shin, M. Jung, J. Lee, C. Park and S. Kang, Appl. Surf. Sci., 2016, 367, 147-152.

138 X. Li, Y. Yang, L. Liu, Y. Chen, M. Chu, H. Sun, W. Shan and Y. Chen, Adv. Mater. Interfaces, 2020, 7, 1-10. 
139 A. M. Alahmari, S. Darwish and N. Ahmed, Int. J. Adv. Des. Manuf. Technol., 2016, 86, 2411-2431.

140 H. Chen, X. Zhang, L. Ma, D. Che, D. Zhang and T. S. Sudarshan, Appl. Surf. Sci., 2014, 316, 124-131.

141 J. A. McCoy, R. L. McEntaffer and D. M. Miles, Astrophys. J., 2020, 891, 114.

142 X. Wang, W. Xu, L. Liu, Z. Zhang, M. Jin and M. Kang, Appl. Phys. A: Mater. Sci. Process., 2017, 123, 592.

143 A. Gruner, L. Naumann, J. Schille and U. Loeschner, J. Laser Micro/Nanoeng., 2019, 14, 190-197.

144 G. D. Bixler and B. Bhushan, J. Colloid Interface Sci., 2013, 393, 384-396.

145 T. Wang, Y. Wan, Z. Kou, Y. Cai, B. Wang and Z. Liu, Proc. Inst. Mech. Eng., Part H, 2016, 230, 1086-1095.

146 L. Ma, I. Howard, M. Pang, Z. Wang and J. Su, Micromachines, 2020, 11, 1-13.

147 R. Kurniawan, S. Ali and T. J. Ko, Measurement, 2020, 160, 107784.

148 G. Xiao, Y. He, Y. Huang and Q. Li, IEEE Access, 2019, 7, 107635-107647.

149 C. Liu, L. Zhu, J. Li and Y. Liang, Microsc. Res. Tech., 2019, 82, 615-623.

$150 \mathrm{~W}$. Liu, H. Ni, P. Wang and Y. Zhou, Beilstein J. Nanotechnol., 2020, 11, 24-40.

151 Y. Li, Z. Cui, Q. Zhu and S. Narasimalu, Coatings, 2020, $\mathbf{1 0}(4), 337$.

152 X. Su, D. Hao, Z. Li, X. Guo and L. Jiang, J. Mater. Chem. B, 2019, 7, 1322-1332.

153 C. Cao, B. Yi, J. Zhang, C. Hou, Z. Wang, G. Lu, X. Huang and X. Yao, Chem. Eng. J., 2020, 392, 1385-8947.

154 E. Liu, L. Li, G. Wang, Z. Zeng, W. Zhao and Q. Xue, Sci. Rep., 2017, 7, 1-9.

155 C. C. Büttner and U. Schulz, Adv. Eng. Mater., 2011, 13, 288295.

156 J. W. Gose, K. Golovin, M. Boban, J. M. Mabry, A. Tuteja, M. Perlin and S. L. Ceccio, J. Fluid Mech., 2018, 845, 560580.

157 Q. An, B. Zhang, G. Liu, W. Yang, H. Zhao, J. Wang and L. Wang, J. Taiwan Inst. Chem. Eng., 2019, 97, 389-396.

158 C. Guo, Q. Tian, H. Wang, J. Sun, L. Du, M. Wang and D. Zhao, Micro Nano Lett., 2017, 12, 439-444.

159 T. Tamura, R. Akiyama, R. ichiro Tanaka, H. Kawamoto and S. Umezu, J. Food Eng., 2020, 277, 109919.

160 I. González-Neria, A. Alonzo-Garcia, S. A. MartínezDelgadillo, V. X. Mendoza-Escamilla, J. A. Yáñez-Varela, P. G. Verdin and G. Rivadeneyra-Romero, Chem. Eng. J., 2019, 374, 1138-1152.

161 Y. Luo, D. Zhang, Y. Liu and E. Y. K. Ng, J. Mech. Med. Boil., 2016, 16, 1-15.

162 X. Chu, X. Zeng, W. Zhuang, W. Zhou, X. Quan and T. Fu, J. Manuf. Process., 2019, 44, 418-426.

163 Y. Liu, H. Gu, Y. Jia, J. Liu, H. Zhang, R. Wang, B. Zhang, H. Zhang and Q. Zhang, Chem. Eng. J., 2019, 356, 318-328.

164 A. Gaddam, A. Prabhakaran, A. Agrawal and S. S. Joshi, J. Micromech. Microeng., 2020, 0-27.

165 G. A. Zampogna, J. Magnaudet and A. Bottaro, J. Fluid Mech., 2019, 858, 407-436.
166 Z. Wang, L. Xu, X. Wu and J. Chen, Microsyst. Nanoeng., 2018, 4, 1-8.

167 W. Bing, L. Tian, Y. Wang, H. Jin, L. Ren and S. Dong, Adv. Mater. Technol., 2019, 4, 1-9.

168 Z. Li and Z. Guo, Nanoscale, 2019, 11, 22636-22663.

169 H. Chen, X. Zhang, D. Che, D. Zhang, X. Li and Y. Li, Adv. Mech. Eng., 2014, 10, 425701.

170 H. Chen, D. Che, X. Zhang and D. Zhang, J. Appl. Polym. Sci., 2015, 132, 1-8.

171 Y. Luo, Y. Liu and D. Y. Zhang, Surf. Rev. Lett., 2015, 22, 112.

172 Y. Luo and D. Zhang, Appl. Surf. Sci., 2013, 282, 370-375.

173 M. Liu, S. Li, Z. Wu, K. Zhang, S. Wang and X. Liang, Eur. J. Mech. B/Fluids, 2019, 77, 87-97.

174 W. Dai, M. Alkahtani, P. R. Hemmer and H. Liang, Friction, 2018, 7, 603-612.

175 J. Tiainen, A. Grönman, A. Jaatinen-Värri and L. Pyy, Renewable Energy, 2020, 155, 79-89.

176 H. Wu, Y. Jiao, C. Zhang, C. Chen, L. Yang, J. Li, J. Ni, Y. Zhang, C. Li, Y. Zhang, S. Jiang, S. Zhu, Y. Hu, D. Wu and J. Chu, Nanoscale, 2019, 11, 4803-4810.

177 L. P. Chamorro, R. E. A. Arndt and F. Sotiropoulos, Renewable Energy, 2013, 50, 1095-1105.

178 J. Wang, C. Song, Z. Zhong, Z. Hu, S. Han, W. Xu, J. Peng, L. Ying, J. Wang and Y. Cao, J. Mater. Chem. C, 2017, 5, 5005-5009.

179 T. Singh and A. Dvivedi, Int. J. Mach. Tools Manuf., 2016, 105, 1-13.

180 S. S. Anasane and B. Bhattacharyya, J. Manuf. Process., 2017, 28, 285-294.

181 J. Man, H. Yang, Y. Wang, H. Chen and F. Xiong, Opt. Laser Technol., 2019, 119, 105669.

182 K. Koeltzsch, A. Dinkelacker and R. Grundmann, Exp. Fluids, 2002, 33, 346-350.

183 D. Wu, J. J. Wang, G. Y. Cui and C. Pan, Sci. China: Technol. Sci., 2020, 63, 214-222.

184 G. Hirt and M. Thome, Prod. Eng., 2007, 1, 351-356.

185 Y. Lu, W. Luo, X. Wu, B. Xu, C. Wang, J. Li and L. Li, Polymers, 2020, 12(6), 1307.

186 J. Bai, F. Tang, X. Xiong, S. He, J. Wang and X. Wang, Int. J. Adv. Des. Manuf. Technol., 2019, 103, 4553-4562.

187 J. Yan, Z. Zhang, T. Kuriyagawa and H. Gonda, Int. J. Adv. Des. Manuf. Technol., 2010, 51, 957-964.

188 W. Natsu, T. Ikeda and M. Kunieda, Precis. Eng., 2007, 31, 33-39.

189 W. Abu Rowin, J. Hou and S. Ghaemi, Exp. Therm. Fluid Sci., 2018, 94, 192-204.

190 Y. Li, K. Zhou, X. Zhao and Q. Kong, J. Micromech. Microeng., 2017, 27, 0115001.

191 G. Liu, M. Wang, L. Xu, A. Incecik, M. A. Sotelo, Z. Li and W. Li, Appl. Ocean Res., 2020, 99, 102142.

$192 \mathrm{~J} . \mathrm{Yu}, \mathrm{Z} . \mathrm{Su}, \mathrm{Z}$. Wu and M. Tan, IEEE Trans. Ind. Electron., 2016, 63, 3108-3116.

193 Z. Wu, J. Yu, J. Yuan and M. Tan, IEEE ASME Trans. Mechatron., 2019, 24, 260-270.

194 A. R. Chowdhury, W. Xue, M. R. Behera and S. K. Panda, J. Mar. Sci. Technol., 2016, 21, 102-114. 
195 L. Chunbao, L. Li, L. Yulong, L. Changsuo and Z. Yubo, Appl. Bionics Biomech., 2016, 2016, 3641651.

196 C. C. Büttner and U. Schulz, Smart Mater. Struct., 2011, 20, 094016.

197 Y. Luo, Y. Liu and D. Y. Zhang, Eur. J. Environ. Civ. Eng., 2015, 19, 931-949.

198 L. Xi, Phys. Fluids, 2019, 31, 1-43.

199 E. J. Soares, J. Nonnewton. Fluid Mech., 2020, 276, 104225.

200 A. V. Singh, A. Rahman, N. V. G. Sudhir Kumar, A. S. Aditi, M. Galluzzi, S. Bovio, S. Barozzi, E. Montani and D. Parazzoli, Mater. Des., 2012, 36, 829-839.

201 R. Stefani, Significance, 2012, 9, 13-17.

202 Y. Wang, N. Li, Y. Ma, J. Tong, W. Pfleging and J. Sun, Soil Tillage Res., 2020, 196, 104432.

203 J. Wang, T. Nakata and H. Liu, Biomimetics, 2019, 4, 1-19.
204 Z. Pang, H. Zhou, G. Xie, D. Cong, C. Meng and L. Ren, Opt. Laser Technol., 2015, 70, 89-93.

205 T. Ibatan, M. S. Uddin and M. A. K. Chowdhury, Surf. Coat. Technol., 2015, 272, 102-120.

206 C. Huang and Z. Guo, J. Bionic Eng., 2019, 16, 769-793.

207 H. Y. Yu, H. C. Zhang, Y. Y. Guo, H. P. Tan, Y. Li and G. N. Xie, Continuum Mech. Thermodyn., 2016, 28, 13611371.

208 G. Huang, Y. Zhu and Z.-Y. Liao, Geophys. Res. Lett., 2020, 031.

209 L. Li, J. Wang, T. Li, W. Song and G. Zhang, J. Appl. Phys., 2015, 117, 104308.

210 X. Chang, L. Li, T. Li, D. Zhou and G. Zhang, RSC Adv., 2016, 6, 87213-87220.

211 H. J. Park, D. Saito, Y. Tasaka and Y. Murai, Exp. Therm. Fluid Sci., 2019, 109, 109919. 